Vol. 10, n² | 2006

Varia

\title{
Historiographie du crime et de la justice criminelle dans l'espace français (1990-2005)
}

Partie II : de la Révolution au XXI ${ }^{\mathrm{e}}$ siècle

Xavier Rousseaux

\section{OpenEdition}

\section{Journals}

Édition électronique

URL : https://journals.openedition.org/chs/224

DOI : $10.4000 /$ chs.224

ISSN : 1663-4837

\section{Éditeur}

Librairie Droz

\section{Édition imprimée}

Date de publication : 1 décembre 2006

Pagination : 123-161

ISBN : 978-2-600-01129-7

ISSN : 1422-0857

\section{Référence électronique}

Xavier Rousseaux, « Historiographie du crime et de la justice criminelle dans l'espace francais

(1990-2005) », Crime, Histoire \& Sociétés / Crime, History \& Societies [En ligne], Vol. 10, n² | 2006, mis en ligne le 01 janvier 2010, consulté le 22 mars 2022. URL : http://journals.openedition.org/chs/224 DOI : https://doi.org/10.4000/chs.224

Ce document a été généré automatiquement le 22 mars 2022.

(C) Droz 


\title{
Historiographie du crime et de la justice criminelle dans l'espace français (1990-2005)
}

Partie II : de la Révolution au XXI ${ }^{\mathrm{e}}$ siècle

\author{
Xavier Rousseaux
}

\section{NOTE DE L'ÉDITEUR}

Initialement, cette contribution à la journée organisée par Mario Sbriccoli à Ferrare couvrait la période 1990-2003. Dans la publication, nous avons étendu le bilan aux publications parues jusqu'en 2005.

1 Pour la France contemporaine, René Lévy notait en 1996, l'hétérogénéité des recherches d'histoire du crime produites depuis le milieu des années 1980. Il en soulignait les raisons d'ordres divers: approche peu critique des sources, absence de dialogue entre deux pans de la corporation historienne: historiens de lettres et historiens du droit, faible spécialisation du domaine, difficultés de publication ${ }^{1}$. Près d'une décennie plus tard, l'évolution est nette. Les deux derniers siècles ont fait l'objet de recherches significatives, entendons des monographies issues le plus souvent de thèses de doctorat, et connaissent une animation scientifique permanente sous forme de colloques et de bilans. La synthèse la plus considérable et la plus récente est le travail de Jean-Claude Farcy sur deux siècles d'histoire de la justice en France, mais il faut citer également les travaux de Lévy, Audren, Petit et Chauvaud et le colloque sur Histoire et archives judiciaires ${ }^{2}$.

2 Apports et lacunes ont été analysés sur base d'une enquête historiographique quasi exhaustive jusqu'en 2000, par Jean-Claude Farcy. Nous renvoyons à ses principales conclusions et dans cette partie n'avons sélectionné que quelques travaux significatifs et récents dans le domaine. Notant un repli des recherches sur les pratiques des tribunaux dans le long $\mathrm{XIX}^{\mathrm{e}}$ siècle, l'absence de travaux sur la justice ordinaire du $\mathrm{XX}^{\mathrm{e}}$ 
siècle, le reflux des études sur la prison, une prosopographie focalisée sur les élites, la surreprésentation, bicentenaire oblige, des recherches sur la justice "intermédiaire ", l'auteur rappelle que l'engouement des études contemporaines est nourri par les préoccupations actuelles du rapport de la société française à sa justice ${ }^{3}$.

\section{Crime et révolution judiciaire, de la fin de l'Ancien Régime à l'Empire}

3 Dès les soubresauts de la Révolution, la question pénale est au centre des critiques et des réformes. On a vu que le récent programme de recherche sur les officiers moyens a mis en lumière le phénomène de distanciation progressive du monde judiciaire vis-àvis de la source de toute justice, le Roi ${ }^{4}$. Une telle analyse remet en perspective longue le phénomène bien connu d'engagement des procureurs de justice et des avocats «sans cause » dans le déclenchement de la Révolution. Sans eux la critique de la justice d'Ancien Régime n'aurait sans doute pas abouti à l'entreprise la plus systématique de rationalisation de l'appareil d'État. Plaidant pour accorder plus d'attention «au rôle social de la fiction dans notre approche du passé ", Sara Maza s'intéresse aux mémoires des avocats décrivant les grandes affaires de la fin de l'Ancien Régime. Dans une société sans jury populaire, à qui s'adressaient ces textes, contribuant "puissamment à faire progresser l'idée d'une nécessaire réforme du système judiciaire»? "Et comme le pouvoir judiciaire constituait le fondement du pouvoir monarchique en France, ces mémoires appelant "l'opinion publique» à remplir une fonction qui revenait autrefois au roi seul ne pouvaient qu'avoir un impact politique de plus en plus fort $~^{5}$. Au terme d'une démonstration serrée, l'auteur conclut à la contribution des causes célèbres à la révolution de la culture politique de la cour royale vers l'assemblée élective et représentative : «l'impact de ces grandes affaires des années 1770 et 1780 sur le public, la visibilité et l'ambition croissante des avocats engagés dans ces affaires et le succès de plus en plus large des écrits qui s'y rapportaient, tout contribue à faire ressortir les perceptions changeantes de la sphère publique française au XVIIIe siècle $»^{6}$. Si le rôle des jeunes avocats d'Ancien Régime, Mirabeau, Condorcet, Desmoulins en Révolution est connu, l'activité des juges en revanche souffrait du discrédit porté sur eux par les pamphlets et les cahiers de doléances.

\section{La justice révolutionnaire : éclairée ou réactionnaire?}

On a pu déplorer que le bicentenaire de la Révolution n'ait guère consacré de place aux travaux sur le crime et la justice, pas plus qu'aux recherches juridiques par ailleurs. La recherche sur cette période doit beaucoup au Centre d'histoire judiciaire, alors dirigé par Jean-Pierre Royer et Renée Martinage. Après des travaux sur le jury criminel, vint une série de colloques sur la première république, le juge de paix, le développement du modèle français en Belgique puis dans l'ensemble des pays sous influence révolutionnaire et impériale ${ }^{7}$. Ces travaux poursuivis par des thèses et menés en parallèle avec nos propres recherches tentaient une approche croisée à plusieurs niveaux de la Révolution pénale. Saisir la justice nouvelle dans son articulation: de la production normative au système pénal en passant par la mise en place de la magistrature, la poursuite des crimes et le jugement des délinquants. L'établir à partir du centre (Paris) et des pratiques des différents pays (départements, belges, rhénans, italiens). Tenter de relier la révolution pénale à la construction étatique libérale sous le 
Directoire, sécuritaire sous le Consulat et l'Empire. Le second volet de cette recherche visait à approfondir la thématique tout en la départementalisant

à l'échelle du département de Sambre-et-Meuse ${ }^{8}$. En matière pénale, de nouvelles instances comme la justice municipale et la simple police, la justice correctionnelle et la justice d'appel de la Révolution et de l'Empire sont des chantiers ouverts. Les justices d'exception, en particulier la justice militaire, laquelle ne se limite pas à juger des militaires, ou la justice maritime commencent à faire l'objet de travaux.

Il en va de même du parquet révolutionnaire qui fait l'objet d'un chapitre de l'histoire du parquet ainsi qu'une relecture juridique de la Terreur judiciaire. Éric de Mari pointe la difficulté d'étudier le parquet « de la période monarchiste, terroriste et directoriale ». La question centrale est celle du contrôle du parquet. Nommés par le roi, mais «à vie », les commissaires du roi sont privés du droit d'accusation et cantonnés dans celui du contrôle de l'application des lois. En 1791, le droit devient l'affaire de tous, car citoyens, juges de paix et directeurs de jurys élus peuvent concourir à la mise en accusation. Discrédités par leur pratique, les commissaires sont supprimés à l'avènement de la Terreur. La remise en cause de l'organisation duale et équilibrée sanctionne

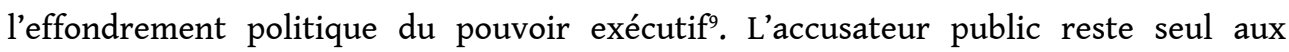
commandes et développe lors de la «mise hors la loi » des compétences qui vont jusqu'à la rédaction des actes d'accusation. Les accusateurs publics restent cependant les premières victimes des épurations politiques. Parent pauvre de l'historiographie révolutionnaire, le Directoire offre pourtant de larges perspectives au chercheur. Le retour à la dualité est assuré. Les commissaires révocables sont bientôt dotés de substituts. Instituée à force de rapports et d'états, la hiérarchie structure peu à peu le ministère public. Le commissaire est «l'œil du pouvoir fixé sur l'administration judiciaire, il est l'esquisse des grands traits du ministère public moderne ${ }^{10}$. L'accusateur public en revanche est en quelque sorte condamné. Très isolé, élu et représentant « d'une caste de républicains fortunés", confiné dans la surveillance des juges de paix, il devient "un simple rouage de l'État ». Avec l'avènement de la fonctionnarisation de l'État, le ministère public ne tardera pas à rentrer dans le rang. Il devient désormais un instrument de pouvoir, « corollaire du triomphe du pouvoir exécutif " ${ }^{11}$.

6 Dans l'historiographie française de la Révolution, le point d'appui comme l'échelle d'observation choisie conditionne des interprétations souvent engluées dans le jugement moral porté sur les années révolutionnaires. "La Terreur» symbolise ce débat en particulier la terreur judiciaire, revisitée par Patrick Gueniffey dans la ligne de François Furet. Faut-il interpréter la loi de Prairial comme une loi "réactionnaire » « prototype d'une juridiction totalitaire $»^{12}$, ou une fatalité inhérente à toute révolution considérée comme une modalité de changement ${ }^{13}$ ? Quoiqu'inscrite dans cette polémique, l'analyse qu'effectue Éric de Mari à propos de la loi de Prairial a le mérite de montrer que rien n'est presque dit sur la formation, la justification et les pratiques de la justice d'exception révolutionnaire, en particulier les politiques judiciaires de la Terreur, et la pratique des justices militaires du Directoire et des régimes qui lui succédèrent ${ }^{14}$.

7 La justice criminelle, clé de voûte de la nouvelle justice de la Révolution est éclairée par la recherche de Robert Allen sur les tribunaux d'une quinzaine de départements, de 1792 à 1811. À partir du fonctionnement quotidien des justices ordinaires, il met en évidence combien le projet de l'Assemblée constituante se comprend par rapport à la justice d'Ancien Régime. Les Constituants ont «ouvert les institutions de la justice à 
l'influence des simples citoyens $»^{15}$. Au risque de voir la justice prendre une autre tournure que ne le voulaient les réformateurs. En matière politique, les nombreux acquittements prononcés par les tribunaux criminels montrent que la politique criminelle du gouvernement et les préoccupations des jurés différaient franchement. L'institution des jurés devenait un lieu d'interpénétration d'une culture juridique éclairée et des pratiques culturelles locales. Enfin, l'existence du jury introduisit une véritable culture du débat contradictoire, une démocratie au prétoire, que les gouvernements successifs de Napoléon à Vichy tentèrent de réduire. Cette appréciation nuancée s'oppose à l'interprétation donnée par Howard Brown dans ses récents travaux. Spécialiste de l'armée, ce dernier a davantage exploré la piste des justices d'exception militaires. Instruments d'un renforcement de l'exécutif motivé par la mise au pas de la violence révolutionnaire, les tribunaux militaires contribuèrent pour lui à la création d'un « État sécuritaire ".

8 Le débat rebondit récemment avec le dernier ouvrage de Jean-Clément Martin consacré à la question de la violence dans l'histoire de la Révolution. Dans la ligne des travaux soucieux d'une compréhension raisonnée des usages de la violence dans les sociétés contemporaines, " plus qu'une histoire de la Révolution et des violences, c'est une lecture de la Révolution par la violence» que propose Violence et Révolution. On peut résumer cette lecture de la décennie révolutionnaire (1789-1799) en quatre moments-clés. "La violence, telle qu'elle structurait les équilibres de la société française, a été d'abord une des raisons du déclenchement de la Révolution; elle a acquis ensuite une autonomie dans l'ensemble $d u$ corps social; elle a enfin servi de moyen d'intervention et de manipulation dans les débats politiques, pour devenir à la fin de la période une réalité intégrée dans une gestion politique de l'État ${ }^{16}$. Cet essai, à rebours des lectures justificatrices ou disqualifiantes de la violence dans la Révolution donne une importance-clé aux pratiques de la justice.

Reste à renouveler dans la même veine, l'histoire de l'organisation judiciaire, normative et pénale de 1810-1811 et les pratiques du Grand Empire, encore peu connues, malgré leur caractère fondateur pour de nombreux États-nations européens.

\section{La pénalisation de l'enfermement}

Bien que progressivement développée dans le dernier siècle de l'Ancien Régime, la domination de la privation de la liberté comme sanction pénale s'exprime au tournant du XIX siècle en Europe. La Révolution et l'Empire donneront à la France un cadre juridique et une structure administrative étatique à l'enfermement.

11 Les diverses formes d'enfermement pénal ont été sensiblement explorées sous l'impulsion de Michelle Perrot et grâce au souffle intellectuel de Surveiller et punir de Michel Foucault et du temps des prisons de Pierre Deyon ${ }^{17}$. "Cette histoire s'inscrit dans le temps long des mentalités et de l'organisation structurelle : le travail forcé des pauvres, dans un lieu fermé (....). Ce processus, à l'œuvre depuis le XVI siècle en Europe, ne fait que continuer en se développant au XIXe siècle ${ }^{18}$. Cette histoire est jalonnée, par les grandes thèses d'André Zysberg sur les galériens du Roi-Soleil, Jacques-Guy Petit sur la naissance de la prison (1770-1870), Jean-Claude Vimont sur la prison politique au XIX ${ }^{\mathrm{e}}$ siècle, et les bagnes d'Outremer ${ }^{19}$. Comparant les projets idéalistes des Lumières avec la réalité du système pénitentiaire du premier $\mathrm{XIX}^{\mathrm{e}}$ siècle - la prison réelle et la prison imaginaire -, J.G. Petit souligne "qu'en développant la prison pénale, l'État moderne peut accentuer la répression des menus délits et la surveillance des classes pauvres avec un appareil de supplices 
d'autant plus efficace qu'il est caché $\aleph^{20}$. Son moteur principal est l'entreprise générale qui organise le travail pénitentiaire. Son public est essentiellement composé de jeunes célibataires, journaliers de la campagne. «La prison fonctionne, pour les jeunes migrants qui quittent leur campagne autour de 1840, au moment de la première vague de l'exode rural, à la fois comme un pôle de répulsion et un abcès de fixation $»^{21}$. L'histoire de la prison est celle de l'émergence d'une administration pénitentiaire qui dut se défaire successivement, «des philanthropes et de leurs alliés du corps médical, des prêtres et plus difficilement des entrepreneurs généraux (...). La mise en entreprise du travail condamne toute tentative de réforme par l'administration. Butant sur le travail, "les réformes carcérales sont contemporaines de ses créations et recréations ". Enfin la surveillance policière sur les condamnés libérés est l'autre versant du système, permettant la réalimentation des prisons. Globalement jusqu'en 1840, bienfaisance et répression ont partie liée car chez les philanthropes «on crut pouvoir résoudre la question sociale par la réforme pénitentiaire $»^{22}$.

Depuis «ces peines obscures", quelques recherches ont été consacrées à des populations particulières de détenus: les femmes détenues ou bagnardes ${ }^{23}$, les politique ${ }^{24}$ et une appréciation globale de la prison républicaine ${ }^{25}$ dans un courant historiographique international. La période 1750-1850 demeure globalement dans l'ombre jusqu'à ce que tout récemment de nouveaux travaux éclairent certains aspects méconnus comme le bagne. Entre la fin des galères (1748) et l'arrimage de la prison au système pénal (1811), les bagnes constituent le chaînon manquant, celui qui transforme les galériens en forçats.

L'exploitation des bagnards, le travail forcé par la Marine, est au cœur de la recherche de Frédérique Joannic-Seta. Dans la seconde moitié du XVIII ${ }^{\mathrm{e}}$ siècle, " période de réflexion sur la pénalité ", le bagne généralise sur le territoire (de Brest, Lorient, Rochefort et Toulon) trois idées pénales des Lumières. L'exemplarité de la peine signifiée par le passage de la chaîne, la rédemption par le travail, tout au moins pour les forçats guérissables, et l'utilité de la peine pour la société, à travers le travail pour la Marine ${ }^{26}$. Pour l'auteur, «lieu de trafic et de transgression» durant la Révolution, le bagne ne put cependant constituer une alternative crédible à la prison pour les réformateurs. On peut en dire autant du passage de la chaîne, rétabli de 1792 à 1836, à travers les régions françaises. Il constitue à la fois un des derniers feux de l'économie des supplices et rappelle les hésitations pénales des temps révolutionnaires ${ }^{27}$. Curieusement, la prison révolutionnaire et impériale reste dans l'ombre. Les études menées sur les départements belges et hollandais montrent cependant que sous le Directoire, la départementalisation des prisons est une catastrophe. En revanche, sous le Consulat, la « centrale » devient le point névralgique du système pénitentiaire. Un modèle qui se nourrit à la confrontation à d'autres modèles d'enfermement pénal : celui des Pays-Bas autrichiens symbolisé par la maison de correction de Gand et l'entreprise de Lieven Bauwens; et plus tard, le modèle hollandais, héritier des Rasphuizen du XVI ${ }^{\mathrm{e}}$ siècle $^{28}$. Un modèle promis à un bel avenir aux $\mathrm{XIX}^{\mathrm{e}}$ et $\mathrm{XX}^{\mathrm{e}}$ siècles, siècles par excellence des archipels carcéraux.

\section{Justice et criminalité au $\mathrm{XIX} \mathrm{X}^{\mathrm{e}}-\mathrm{XX}^{\mathrm{e}}$ siècle}

Si la césure $\mathrm{XIX}^{\mathrm{e}}-\mathrm{XX} \mathrm{e}^{\mathrm{e}}$ siècle est, par de nombreux aspects, artificielle pour l'histoire judiciaire et criminelle, elle conditionne encore la production scientifique française, 
fortement articulée sur la dynamique des régimes politiques. Nous conserverons la distinction pour dégager les principaux champs spécifiquement labourés pour les périodes 1815-1914 et 1914-2005.

Dans la foulée de leurs collègues modernistes et médiévistes, les chercheurs se sont interrogés sur leurs sources, en particulier les archives judiciaires ${ }^{29}$. À partir de 1800 , l'énorme masse documentaire, la précision croissante des archives n'a d'égal que leurs difficultés d'interprétation. Susceptibles de lectures diverses, ces archives permettent d'étudier à la fois selon la typologie de Jean-Claude Farcy l'institution judiciaire et le pouvoir d'État, les rapports entre institutions et population, et autorisent de nombreuses plongées d'histoire économique, politique ou sociale ${ }^{30}$. Certaines périodes noires de l'histoire française, Vichy, la guerre d'Algérie et dans une moindre mesure la première guerre ont suscité des débats de société virulents et conduits de nombreux chercheurs à affronter les fourches caudines de la «dérogation » aux interdictions de consultation, avec des résultats souvent sensationnels, mais peu contrôlables par la communauté historienne. En outre, la fascination pour les archives du crime et de la régulation sociale fait parfois oublier aux chercheurs les pièges et le formatage de ces sources.

Par rapport aux travaux précédents fondés essentiellement sur des études de criminalité d'une part et des recherches sur l'institution judiciaire (police, justice et prison) de l'autre, une innovation récente est la redécouverte de la place du "crime " dans l'histoire culturelle, intellectuelle et des sciences humaines. Le colloque sur l'histoire de la criminologie française coordonné par Laurent Mucchielli ${ }^{31}$ balisait cette autre perspective d'interprétation des archives du crime. Mais d'autres travaux, notamment ceux des historiens américains, souvent portés à privilégier l'analyse des discours sur le dépouillement des archives, annonçaient ce «tournant linguistique du crime». Personne plus que Michelle Perrot ne pouvait symboliser cette double approche sur les réalités institutionnelles et les représentations collectives, que l'on retrouve au cœur d'un recueil d'articles fondateurs ${ }^{32}$.

\section{De l'encre sur le sang : les discours sur le crime}

\section{Imaginaires experts du crime}

17 Frédéric Chauvaud s'est intéressé à une catégorie d'acteurs de la justice, jusque-là, méconnus : les experts. La démarche est de "privilégier l'essor d'une corporation qui n'est pas celle des médecins $»^{33}$. Membres relégués en marge "de la société judiciaire», les experts depuis 1791 sont pourtant des acteurs importants du procès. Leur mission couvre «deux domaines bien distincts, celui de l'esprit et celui du corps». Essentiellement focalisée autour de l'aliénation mentale et aiguillée par la question judiciaire de la responsabilité individuelle, l'expertise du XIX ${ }^{e}$ siècle est d'abord médico-légale. Dans le domaine corporel, l'expertise s'intéresse "aux lésions et blessures graves ainsi qu'aux cadavres ${ }^{34}$. Armé de la notion de compétence, le monde des experts se professionnalise vers les années 1850 au point d'apparaître aux yeux de certains observateurs comme les véritables juges dictant la sentence. Si l'axe du procès se déplace au cours du XIX ${ }^{e}$ siècle, «le fait l'emporte sur le droit, l'expert sur le juge » c'est que l'expertise judiciaire se déplace de plus en plus vers l'enquête judiciaire c'est-à-dire, l'instruction, le parquet et la police, comme le montre l'exemple de la dactyloscopie. L'expertise se mue en police scientifique ou criminalistique vers 1909. Néanmoins cette expertise reste 
essentiellement liée à la preuve du crime; elle demeure donc liée à l'accusation. Le statut de l'expertise se transforme également sous l'influence de cette évolution. De preuve testimoniale (la conviction de l'expert, elle devient preuve scientifique (la matérialité des traces ${ }^{35}$ ). La première conception est celle de la médecine aliéniste, la seconde de la police scientifique. Enfin l'auteur conclut son opus en rappelant qu'à la veille de 1914, l'expertise fascine les spécialistes du crime par son pouvoir apparent de livrer, «à la manière d'une clé universelle, la vérité du crime ».

Autre regard sur "la vérité du crime», celui de Marc Renneville tentant de saisir les liens entre représentation du crime et de la folie. Au point de départ, la conception de la folie sous l'Ancien Régime, figure de déraison totale et incurable: la "folie criminelle ». Au cours de deux siècles, cette folie criminelle s'est transformée en " folie du crime » déraison partielle qui conduit au crime. Derrière cette transformation se cache une double acculturation entre deux disciplines: médecine et droit, dont témoigne l'expertise psychiatrique.

Dès le début du XIX ${ }^{e}$ siècle, la phrénologie se targuait d'expliquer le crime sans mobile apparent par la «bosse » du crâne. L'auteur rendait ces lettres de noblesse à cette théorie, mue par l'optimisme rationaliste du premier XIXe siècle dans «le langage des crânes $\|^{36}$.

Se résorbant dès les années 1840 , comme le dit joliment Marc Renneville, la théorie de la bosse du crime laisse place à une médicalisation de comportements collectifs : récidivistes, délinquants juvéniles, foules en manifestation ou peuples colonisés. Une « conception laïque du mal » est entérinée par les institutions répressives, conception qui mène au "criminel-né » de Lombroso et à l'incorrigibilité d'une frange criminelle. Au début du $\mathrm{XX}^{\mathrm{e}}$ siècle, les médecins critiquent cette conception de totale déraison et la fausse alternative de la responsabilité: nulle ou totale. Ils décrivent l'aliénation mentale sur un escalier à degrés, et justifient un dispositif de défense sociale. L'entredeux-guerres, grande époque de la défense sociale, se traduit en politiques criminelles comme les thérapies contraintes et la stérilisation des "asociaux", que l'expérience totalitaire ne dissuade pas de promouvoir. Cette période paraît néanmoins moins familière à l'auteur, ou moins porteuse d'interprétations nouvelles. Marc Renneville convoque alors le cinéma comme lieu d'expression de cette culture médico-légale de la folie criminelle. Générateur de nouvelles représentations par la spécificité de ses règles d'écriture, le cinéma a permis aux idées savantes de se frayer un chemin dans l'imaginaire collectif. En représentant l'horreur, en démontant les discours contradictoires sur le criminel en déraison, mais rappelle également Marc Renneville, en distillant l'espoir d'une guérison médicale ${ }^{37}$. Cette partie sur le cinéma montre la difficulté d'arrimer un imaginaire collectif à des pratiques quotidiennes. Dès la deuxième moitié du $\mathrm{XX}^{\mathrm{e}}$ siècle, cet imaginaire change d'échelle, de par l'universalité de la criminologie, il est désormais mondial, comme la défense sociale de l'entre-deuxguerres était occidentale et la réforme de la prison du XVIII ${ }^{e}$ siècle européenne.

\section{Imaginaires sociaux du crime}

21 Le cinéma n'est pas le premier vecteur de première représentation "collective » du crime et de sa sanction. Aux historiens modernistes de s'être penchés sur les « occasionnels ", les canards vendus au pied des gibets. Aux médiévistes d'avoir relu les lettres de pardon comme un premier outil de "vulgarisation » de l'image du crime excusable et de l'unique dépositaire du pardon. Mais la grande époque qui donne aux 
discours du crime une expansion sans limites est bien celle du développement de la presse, à partir du dernier tiers du XIX ${ }^{\mathrm{e}}$ siècle. Presse oligarchique et savante comme la Gazette des tribunaux, puis à partir du milieu du siècle, presse populaire, marquée par l'invention du roman policier (l'affaire Lerouge) en 1864 et l'ère du fait divers (l'affaire Troppmann) en 1869. Se situant à distance des principales théories, moralisatrice ou marxiste qui y voyaient un instrument de démoralisation des classes populaires, ou structuraliste y voyant un thème fixé et invariant, Dominique Kalifa propose une approche historique de la littérature populaire du crime, replaçant l'essor et la variété des récits de crime dans une « révolution culturelle silencieuse, qui vit tout un peuple accéder progressivement à l'alphabétisation et à la lecture $»^{38}$.

Le tournant du vingtième siècle est un " observatoire privilégié où approcher l'état du crime et de ses représentations, l'imaginaire qu'il fascine et les arguments qu'il engendre » rappelle l'auteur ${ }^{39}$. Pour trois raisons la " formidable et spectaculaire envolée de toutes les formes de récits de crimes... », en période d'apogée technique et productive de la presse populaire, "la résorption progressive du récit de crime dans celui de l'enquête (...), entraînant avec elle le triomphe de la figure désormais héroïque de linvestigateur, policier, détective et surtout reporter $\aleph^{40}$. Enfin la montée d'un discours alarmiste et d'une rhétorique sécuritaire, alimentés par les débats sur la rélégation des récidivistes et la psychose des attentats anarchistes. Au terme de l'enquête, deux constats majeurs : « la constitution et la diffusion d'un imaginaire original, celui de l'enquête et de ses procédures $»^{41}$, porté grâce à la révolution du journal par une figure neuve du vieil héros épique, le reporter. D'autre part, un discours de "sécurité publique » cristallisé par la figure de l'apache, parangon de la délinquance juvénile et urbaine. Pour autant Dominique Kalifa rappelle-t-il les limites de l'enquête - et de l'histoire culturelle. L'imaginaire n'est pas le réel, le discours sur le discours prend le risque "d'oublier les acteurs sociaux, de négliger leurs conduites et d'ignorer leurs actes $\aleph^{42}$. Ainsi, une prudente lecture constate que ces récits "témoignaient aussi d'une intolérance accrue du corps social à l'égard d'une violence " objective» de plus en plus déclinante et distante». Ces récits s'inscrivent donc "dans un lent et progressif mouvement de moralisation et de 'civilisation de mours', et disent aussi l'intégration croissante dans la rationalité policée de l'ordre industriel $»^{43}$.

Expérience-limite de cette mixture d'encre et de sang, le "livre des vies coupables" publié et accompagné par Philippe Artières souligne toutes les ambiguïtés d'une autobiographie criminelle, révélée par ces cahiers où se racontent des prisonniers. Issu d'une expérience criminologique originale, celle de Lacassagne, père de l'école de Lyon, qui, obnubilé par la volonté de comprendre le criminel, propose à des prisonniers de mettre leur vie sur papier... « on ne peut comprendre autrement l'expérience du Livre des vies coupables que comme relevant de cette folle ambition: percer le mystère de ce personnage en constituant une sorte d'encyclopédie vivante du crime $»^{44}$. Mais jamais Lacassagne ne publiera ces récits. Ceux-ci lui parurent-ils piégés? « ... ces quelques criminels avaient parfaitement intégré certaines de leurs thèses et les récits qu'ils font de leur vie en sont truffés. De la rencontre de ces deux imaginaires était né un genre inclassable, les récits qui échappent ${ }^{45}$. Comment présenter ces récits se demande l'auteur ? L'histoire du rapport de l'auteur avec l'un de ses criminels Charles Double (sic) ${ }^{46}$ montre les limites de la volonté d'expliquer. "Capter les émotions, saisir ce que Foucault appelait si justement 'le marmonnement du monde', 'trouver la bonne distance' de son objet, sans essayer d'en faire un objet d'analyse, de s'identifier à lui, voilà le travail de l'historien $»^{47}$. 
24 Du discours savant au discours criminel en passant par le discours populaire, les recherches récentes ont montré l'importance des facteurs culturels dans la construction de l'image du crime et de son traitement social par la justice ${ }^{48}$. Mais l'historien rappelle que tout discours est situé, et que son producteur a autant d'importance que son récit. Représentations collectives du crime, fascination pour les "grandes affaires criminelles» et pratiques sociales de la justice participent d'une logique commune: celle d'une histoire socio-culturelle du crime, démarche résumée par Dominique Kalifa dans son essai Crime et culture en France au XIX ${ }^{e}$ siècle ${ }^{49}$.

L'obsession du crime pour la société du XIX ${ }^{\mathrm{e}}$ siècle entretient « d'étroites relations avec la culture ». Les affaires criminelles célèbres engendrent de nombreuses productions culturelles. Produit social, le crime ne se définit qu'en fonction des « règles, des valeurs et des sentiments collectifs que se donne une société ». Enfin, réalité incompréhensible, le crime fait l'objet de multiples démarches d'enquête destinées à le transformer en objet lisible et maîtrisable par les différents groupes qui composent la société.

\section{Les pratiques des tribunaux}

Autre voie plus classique, les recherches sur l'activité d'une institution ou sur la figure d'un conflit. Au niveau le plus global, prendre le fonctionnement de la machine judiciaire comme un ensemble est la démarche de Gilles Rouet. Consacré à l'analyse de la justice civile, il s'appuie sur une analyse précise des statistiques judiciaires, utiles à la connaissance de l'État, et profitables aux responsables de cet État ${ }^{50}$. Outre l'aspect de cartographie judiciaire, l'intérêt de son étude réside dans la tentative de replacer le fonctionnement institutionnel des juridictions par rapport aux évolutions de la "demande de justice ", perçue comme révélatrice d'une demande de respect des droits. D'où l'intérêt d'une grande partie des populations du Nord, de l'Est et des villes pour la conciliation. L'auteur souligne le «rôle de l'État » en matière de justice civile. «Durant tout le $\mathrm{XIX}^{e}$ et le $\mathrm{XX}^{e}$ siècles se renforce la régulation étatique » tandis que les tribunaux se fonctionnarisent. Néanmoins la machine judiciaire a mauvaise presse comme si, un immobilisme de la carte judicaire et/ou des institutions impliquerait forcément une pérennité des rapports en juges et justiciables $»^{51}$. Le Droit transposerait alors cette demande de justice en services judiciaires, offerts par l'État.

27 Curieusement cette lecture de l'entreprise judiciaire en termes de besoins sociaux et d'offre de services s'inscrit pour son auteur en opposition aux travaux sur la justice pénale. Or ceux-ci, symbolisés par les travaux reposant sur le compte général de la justice criminelle ${ }^{52}$ évoquent, dans des termes différents (demande de sécurité), les mêmes réalités, prétendument négligées par les historiens. Au delà d'une dénonciation un peu stérile des oppositions entre justice civile et justice pénale, entre sociologues et historiens, c'est sur la nécessité d'une articulation d'ensemble de l'activité judiciaire sur les différents niveaux d'institutions de régulation des conflits qu'invitent ces recherches de sociologie historique de la justice et qui ont attiré l'attention des chercheurs.

28 Au premier niveau, des études récentes ont redécouvert la justice de paix, supprimée depuis 1958. La synthèse dirigée par Jacques-Guy Petit revisite cette institution méconnue qui hante les réflexions actuelles sur la justice de proximité. Création de la révolution, cette justice connaît son apogée dans les années 1860. En phase avec une France rurale, elle offre une triple proximité entre juges et justiciables, symbolisée par 
la conciliation: proximité géographique dans l'horizon du canton, proximité sociale entre les justiciables et les notables locaux, et proximité économique de décisions rapides et peu coûteuses.

L'urbanisation croissante, l'exode rural des plus démunis, mais aussi la professionnalisation croissante de cette justice de proximité en font une institution critiquée et dévaluée à partir du dernier tiers du XIXe siècle, surtout en milieu urbain ${ }^{53}$. Pourtant, habilité à trancher des litiges civils et pénaux, le juge de paix/juge de police décharge les tribunaux d'une masse d'affaires minuscules, qui progressivement envahiront les juridictions supérieures : les tribunaux correctionnels. Les études sur ces derniers ne sont pas légion. Citons la recherche de Nicole Arnaud-Duc sur les jugements de neuf années du tribunal correctionnel d'Aix, laquelle se propose "d'enregistrer et de commenter les grandes tendances des variations d'un tribunal répressif en tentant une approche sociologique du procès pénal et du délinquant jugé $\aleph^{54}$. L'analyse des sources est limitée au jugement mais la saisie se fait sur une période ample : 1825-1905 au prix d'une sélection d'une année sur dix de décisions du tribunal d'Aix. C'est du Code pénal de 1810 que part l'auteur dont la sociologie juridique nourrit l'interrogation des archives. Saisis à travers neuf années, les jugements du tribunal révèlent des évolutions vers une "nouvelle politique pénale, plus attentive à la personne du délinquant et plus ouverte dans sa pratique aux dernières techniques d'investigation $»^{55}$. Évolution des contentieux marquée par la disparition des délits ruraux et forestiers, l'augmentation des condamnations pour vol, et à un niveau bien moindre mais tout de même notable, les affaires de mœurs. À partir de 1860, augmentation de l'ensemble des infractions professionnelles ${ }^{56}$ et déclin de la violence, sauf en 1905. Deux cibles pénales sont privilégiées : les hommes, jeunes et peu insérés, condamnés à l'emprisonnement et à l'amende, les bourgeois, petits commerçants poursuivis pour délits dans leur activité professionnelle et sanctionnés par des amendes. La première bien plus visible et nourrissant le fantasme de la récidive, la seconde manifestant l'inaptitude du code à traiter la délinquance économique et financière. Du point de vue des philosophies pénales, l'auteur remarque que l'utilité sociale de la peine et l'expiation du coupable cèdent le pas à l'écartement de la dangerosité. L'évolution se fait également vers une baisse de la délinquance jugée, en raison d'un taux de classement plus élevé et d'un adoucissement général des peines qui fit parler en 1911 d'une crise de la répression ${ }^{57}$. D'autre part, l'auteur note que la réduction des compétences de cours d'assises à la fin du XIXe siècle met désormais « au centre de l'ordre étatique » le tribunal correctionnel. Enserrant la vie publique dans un réglementarisme de plus en plus précis, celui-ci devient le pivot d'un contrôle social relayé par un travail policier plus actif qui s'attache aux populations inadaptées suspectes de vagabondage ou de vol ${ }^{58}$.

\section{Politiques judiciaires et figures criminelles}

30 Le dernier exemple ouvre d'autre pistes de relecture du crime, celle de la construction de politiques judiciaires focalisées autour de figures criminelles.

31 Au début du XIX ${ }^{e}$ siècle, l'essentiel du contrôle social par les préfets et les juges s'oriente sur la société agraire. Dans une histoire croisée des pratiques et des représentations, appliquant le concept d'économie morale de la foule (E.P. Thompson) à la France du premier XIX ${ }^{e}$ siècle, Nicolas Bourguinat (2002) souligne la contribution du prix du grain à l'instabilité des rapports entre population et État, sous les régimes 
postérieurs à la Révolution. Néanmoins ces rapports se transforment. Dans les années 1840, les émeutes se modernisent ou plutôt le regard de l'État sur elles, surtout durant la monarchie de Juillet qui désormais les politise.

L'approche de Jean-François Wagniart est davantage orientée sur les politiques de la $\mathrm{III}^{\mathrm{e}}$ République face au vagabondage. Elle s'inscrit dans une histoire des représentations, celle "des vagabonds comme exclus et de l'exclusion comme problème $»^{59}$ de la société. Plus particulièrement sur l'enjeu politique et social important du vagabondage dans la grande crise économique de 1873 à 1896. Discours sur le vagabondage, pratiques de prises en charge n'effacent pas pour autant les traces du vagabond et de son errance même biaisées par le discours judiciaire des tribunaux correctionnels. Celui-ci révèle cependant "les hésitations de la justice » entre la construction d'une "classe vagabonde » et la définition d'une catégorie juridique de vagabond. À partir de la fin du XIX siècle, la spécialisation de l'errance s'accompagne d'une identification plus précise des petits délits et des crimes commis par les errants ${ }^{60}$.

Autre catégorie à émerger lentement dans la justice du dix-neuvième siècle : celle de l'enfant à protéger, qui fait l'objet de la belle étude croisant les travaux français, belges, hollandais et québécois ${ }^{61}$. L'originalité de l'ouvrage est de croiser les expériences «nationales» autour des acteurs (les philanthropes) des débats d'idées (les modèles), des mises en pratique institutionnelles (les établissements pour jeunes détenus), puis à la fin du siècle des critiques et des réformes législatives et du renouveau du débat international autour du modèle «protectionnel » de Chicago. Le cas français y paraît singulier durant la $\mathrm{III}^{\mathrm{e}}$ République avec son modèle d'établissement pour jeunes (Mettray) et la résistance des juristes au juge spécialisé du début du $\mathrm{XX}^{\mathrm{e}}$ siècle. Vagabonds, enfants sont réunis par une criminologie de plus en plus conquérante.

La construction du crime comme objet social et politique est au cœur de la période 1885-1914, comme le montre Martine Kaluszynski dans «La République à l'épreuve du crime ». L'investissement de la criminologie scientifique se double d'une volonté d'agir politiquement pour défendre la société contre les menaces. Il en résulte un "paradoxe républicain » fait d'une politique tiraillée entre prévention et exclusion d'une part, et entre amendement et répression, d'autre part ${ }^{62}$. De tels travaux sur les politiques judiciaires ou criminelles contribuent à enrichir progressivement la chronologie des rapports entre populations, État et gouvernements, en soulignant combien la construction des menaces a une histoire ${ }^{63}$.

\section{Violences rurales}

Dans les quinze dernières années, de grandes thèses produites par l'école historique française se sont concentrées sur des crimes particulièrement symboliques. Les violences physiques, surtout mortelles, révélées par les dossiers d'assises ont attiré les chercheurs, particulièrement dans le monde rural.

Dans la veine d'une étude régionale mais orientée sur un crime, se situe l'étude d'Annick Tillier sur les infanticides bretonnes. L'infanticide se trouve « ... À la croisée de multiples territoires (...) l'histoire de la justice et de l'acculturation judiciaire, des paysans, l'histoire des femmes, particulièrement des femmes seules, l'histoire des sensibilités, considérée sous l'angle du sentiment amoureux et du rapport à l'enfant et - enfin - l'anthropologie » ${ }^{64}$. Car c'est autour de la honte et de l'honneur que se joue le théâtre du crime d'infanticide. Annick Tillier utilise le crime comme révélateur de tensions de la société bretonne. 
Tensions entre magistrats, ruraux et jurés dont l'appréciation de la gravité du crime est dissonante. Tensions entre la justice officielle, soucieuse de défendre la famille et punir l'homicide et une justice officieuse, parfois plus féroce, qui peut stigmatiser ou blanchir et qui reconstruit l'unité du village par la mise à l'écart de l'infanticide. Tensions entre les juges et les notables locaux, prêtres et maires. Tensions entre les habitants dans un réseau complexe de hiérarchies : entre femmes mariées et veuves et célibataires, entre hommes et femmes, entre aisés et pauvres, entre exclus et participants au jeu social du mariage. Malgré le caractère construit de l'échantillon, le profil des femmes infanticides est déroutant. La pauvreté n'est pas générale, la misère n'empêche pas la participation aux fêtes, la vie n'est pas uniquement labeur... Et au moment du procès, ces femmes savent se défendre. D'autres aspects de la vie rurale dressent un tableau nuancé de la société bretonne : la rumeur, l'observation des ventres, la mise au ban, l'importance de l'automédication et des guérisseurs, la solitude des femmes de marins, la vulnérabilité des veuves, la rudesse des maîtres, la hantise des célibataires vis-à-vis $\mathrm{du}$ mariage. "Symétrique inversée de l'infanticide » ${ }^{65}$, le parricide fait l'objet d'une magistrale étude de Sylvie Lapalus. Prenant pour objets les coups et blessures et les homicides d'ascendants dans la France du XIX ${ }^{e}$ siècle, l'auteur se livre à une histoire des regards littéraire et journalistique, médical et judiciaire, une histoire de l'incrimination du procès et de la décision et une histoire des conflits révélant en creux les valeurs et les institutions fondamentales pour le $\mathrm{XIX}^{\mathrm{e}}$ siècle français. Le parricide est fréquemment un homme, souvent un rural, généralement un héritier. Manifestation d'un conflit mettant en jeu l'héritage et l'autorité du père de famille, conflit renforcé par les contraintes du Code civil, le parricide est en baisse tout au long du XIX siècle, surtout dans les régions d'émigration et d'industrialisation. Crime « impensable » il fait l'objet d'une "évolution inverse chez les psychiatres et les magistrats : tandis que le premiers mettaient de plus en plus l'accent sur la spécificité de ce crime, les seconds tendaient à le banaliser $»^{66}$. Comme le note Sylvie Lapalus, infanticide et parricide épousent l'évolution complexe de la société du XIX ${ }^{\mathrm{e}}$ siècle. Crimes par excellences, ils ont fini par devenir à la fin du XX $\mathrm{XX}^{\mathrm{e}}$ siècle des circonstances aggravantes de l'homicide dans une société où leur signification sociale cède la place comme symboles de l'horreur absolue aux viols d'enfants et aux crimes contre l'humanité.

Largement ruraux, ces crimes méritent une approche par l'exceptionnel, telle que la pratique Frédéric Chauvaud. Une approche, qui loin d'un mélange de comptages approximatifs et de citations exceptionnelles promeut la (re)construction de l'image du criminel à partir de figures spécifiques. Après avoir consacré une minutieuse étude aux affaires « minuscules » du tribunal correctionnel de Rambouillet, il propose une lecture à plusieurs niveaux des affaires criminelles poitevines autour des «monstres, des désespéré(e)s et des voleurs ». Rappelant que chaque affaire s'inscrit dans un contexte singulier, dans un terroir et un réseau de relations, mais s'efforce de rentrer dans des cadres judiciaires et juridiques: le cadre administratif et judiciaire de la statistique criminelle départementale, le cadre juridique du code pénal, et le cadre intellectuel et discursif d'une construction régionale,- en l'occurrence le Poitou- "parfois instrumentalisée $\aleph^{67}$. Mais ces réalités singulières modifient à leur tour l'image du criminel, en forçant le droit à s'interroger sur la folie, la préméditation, la récidive. Enfin chaque crime, "ramené à l'échelle des individus, appartient à une histoire du paroxysme ». L'analyse du geste des criminels est une occasion d'entrer plus avant dans l'insondable mystère des êtres humains et de mieux comprendre comment fonctionnent les sociétés. Cette interrogation anthropologique invite le chercheur à 
réinvestir les dossiers criminels avec un œil neuf, sur les identités proclamées, les sentiments énoncés, les lieux décrits...

La prégnance des crimes de sang dans le Lot rural examiné par François Ploux lui fait classer cette société parmi les sociétés "à vengeance». Le système vindicatoire s'exerce sous formes de "guerres intercommunales». Dans la ligne des travaux anthropologiques de Claverie et Lamaison, le système de dévolution des biens à l'aîné intervient pour beaucoup dans la pression violente. L'honneur est au cœur d'une société composée d'unités sociales (structurée en segments lignagers ou groupements territoriaux); la cohésion sociale repose sur un affrontement perpétuel entre les différentes unités sociales ${ }^{68}$. La très forte criminalité de sang sous la Restauration est pour l'auteur liée à la violence familiale, accentuée par une pression démographique jusque dans les années 1850 avant que le système se désintègre au prix d'un accroissement de violence sociale. Ploux aborde ensuite la question des rapports entre la société locale et l'appareil judiciaire. On assistait au début du XIX ${ }^{\mathrm{e}}$ siècle à un parasitage de la répression pénale par la pratique vindicatoire. Toutefois cette société est caractérisée par un contrôle tardif de son système de régulation sociale en raison du rôle des notables et de l'existence d'une panoplie de pratiques conciliatoires. La politisation des populations et le clientélisme se superposent à l'existence des groupes anciens, signe de la résistance de cette société aux pratiques nouvelles. Néanmoins le $\mathrm{XIX}^{e}$ siècle est bien le siècle de l'acculturation du Quercy à l'État central, celui où «le système pénal a fini par supplanter le système vindicatoire et les pratiques conciliatoires de résolution du litige qui lui étaient associées ${ }^{69}$.

Politisation et étatisation des pratiques traditionnelles se retrouvent au cœur de l'analyse micro-historique "du dernier des massacres nés de la fureur paysanne", le supplice d'un jeune noble à Hautefaye, en Dordogne, le 16 août $1870^{70}$. Pour comprendre comment a pu se produire ce lynchage, Alain Corbin se livre à une analyse à plusieurs niveaux d'échelle du contexte structurel et conjoncturel.

Ce dernier travail consacre un intérêt renouvelé pour l'histoire de la violence, en particulier rurale. L'Association des ruralistes français y a consacré un colloque et un ouvrage collectif ${ }^{11}$. Les jeunesses rurales ont fait l'objet d'un petit ouvrage de

J.-C. Farcy ${ }^{72}$. À travers des enquêtes sur les conflits familiaux, les querelles de voisinage, la définition des rôles, les travaux des dix-neuviémistes portent sur les liens forts entre communauté rurale et violence. La question de la désaffection croissante des ruraux envers l'usage de la violence aux $\mathrm{XIX}^{\mathrm{e}}$ et $\mathrm{XX}^{\mathrm{e}}$ siècles et les raisons de cette désaffection restent peu explorées. $Y$ répondre suppose d'élargir le débat sur les formes de violence physique ou verbale, par exemple en abordant la place de la violence dans l'éducation scolaire $^{73}$ mais aussi de prendre en compte la vaste "modernisation de la France rurale $»^{74}$. L'histoire de l'insulte politique aux XIX ${ }^{\mathrm{e}}$-XXe siècles par exemple manifeste un double processus: d'extension du champ politique et d'exclusion de certaines catégories d'acteurs individuels et collectifs ${ }^{75}$. Le colloque qui lui a été consacré cherche à la saisir dans ses différents aspects linguistiques, culturels et sociaux mais se termine sur une affirmation non vérifiée: la violence de fait aurait presque toujours été préparée par la violence verbale. Seule l'inscription de la violence verbale dans l'ensemble des comportements «violents» permettrait d'évaluer les théories de "modernisation politique»- l'insulte remplacerait la violence physique ou de "conflits de culture» entre groupes sociopolitiques: l'insulte serait le propre des acteurs éduqués mais serait récupérée dans le folklore par les communautés locales. 
C'est la démarche à laquelle fait penser le titre d'un autre colloque pluridisciplinaire sur « La voix et le geste. Une approche culturelle de la violence sociopolitique». On y devine le fil conducteur d'une conception de la violence comme inhérente à la construction politique. On peut néanmoins regretter que l'approche culturelle fasse l'économie d'une véritable phénoménologie et contextualisation anthropologique et sociale des comportements verbaux ou gestuels ${ }^{76}$.

\section{Menaces urbaines}

Jusqu'ici les déviances et pratiques judiciaires demeurent largement rurales. Poids structurel des masses rurales jusqu'aux années 1940? Effet de sources, mode historiographique ou fascination pour un monde «que nous avons perdu »? Quoi qu'il en soit, avant la seconde guerre, les villes restent largement absentes de ce paysage sinon dans les thèses de doctorat non publiées ou pour des populations spécifiques comme les femmes ${ }^{77}$ ou les homosexuels ${ }^{78}$. Dans ce dernier cas, la construction même du profil repose sur deux éléments : la médiatisation du profil et le contrôle policier, principale source d'information sur des pratiques volontairement discrètes avant les années 1960. Or ce contrôle est particulièrement actif dans les villes où les rencontres d'hommes (en particulier les militaires) attirent l'œil de la police. En l'absence d'une infraction spécifique, les relations homosexuelles entre majeurs sont poursuivies dans le cadre d'infraction comme le vagabondage, traduction légale du «racolage ou de l'outrage public à la pudeur ${ }^{79}$. Indices d'un étiquetage particulier, dû au travail policier ces poursuites sont parfois assumées par les individus concernés; l'étiquetage crée donc des sources utilisables pour l'historien des homosexualités.

L'espace urbain lui-même est le terrain du travail de Simone Delattre, qui s'inscrit dans l'histoire des représentations illustrée par Alain Corbin. En étudiant la nuit à Paris au $\mathrm{XIX}^{\mathrm{e}}$ siècle, elle convoque l'histoire du crime et de la justice dans deux chapitres: contrôle et bas-fonds. L'encadrement policier de la nuit d'une part et les dangers vécus ou perçus d'autre part. Le contrôle de la nuit est affaire de police. "Sur fond d'amélioration globale du dispositif de vigilance nocturne, le sentiment d'insécurité connaît en fait, de la Restauration à la Grande Guerre, d'importantes variations ». La réforme de la police parisienne de 1854 visibilise une logique de cloisonnement social et le reflux des peurs nocturnes vers les quartiers orientaux et marginaux, "non encore domestiqués par le Second Empire $»^{80}$. Le modèle du quadrillage policier rassure plutôt qu'il n'éradique. Néanmoins, l'afflux des pauvres du premier XIXe siècle cède progressivement le pas à une civilisation du centre-ville, "harmonie de fait qui s'instaure, dans les années 1850-1860, entre le réverbère, la rue rectiligne et l'ilôtage policier ». Le vaste assainissement de la ville entrepris sous la monarchie de Juillet se prolonge sous la Troisième République. «Il s'agit d'assigner à chacun une place fixe - l'homme à l'usine, la femme au foyer, l'enfant à l'école, le criminel en prison ${ }^{81}$.

L'expérience de la Commune ravive au bourgeois la peur du peuple dangereux. Le seuil de tolérance s'abaisse, l'ivresse publique inquiète (1873), la dégénérescence hante et conduit à la loi de 1885 sur la relégation des petits voleurs et errants multirécidivistes, consacrant la vocation parisienne dans sa «double fonction de capitale: celle du plaisir et celle du travail $»^{82}$. Les années 1825-1845 (les mieux documentées) sont décisives "elles installent le stéréotype sanglant de l'attaque nocturne " comme fléau citadin endémique. Les classes nocturnes sont rejetées, deviennent dangereuses, et la criminalisation de la nuit 
se manifeste non seulement dans la conception de lieu central de la criminalité mais aussi des exécutions publiques.

\section{Crime et genre} est l'»étonnement suscité par la différence des sexes en matière de délinquance, criminalité, répression pénale et pénitentiaire, en France, et plus largement, dans le monde occidental », différence qui se marque par une présence minime et décroissante des femmes dans la justice pénale aux XIX et $\mathrm{XX}^{\mathrm{e}}$ siècles et détermina un effet direct sur la recherche ${ }^{83}$. De nombreuses recherches ont été consacrées aux femmes délinquantes ou aux délits considérés comme typiquement féminins: infanticide, avortement, empoisonnement, prostitution. point sur la question à travers trois thématiques: l'évolution des illégalismes féminins et de leur représentation, les transformations du traitement pénal, l'accès des femmes à la justice.

illégalismes traduit le repli des crimes sur la famille : après 1850, le rôle féminin de régulation de l'économie morale des subsistances s'atténue comme en témoigne la disparition progressive des infractions publiques impliquant les femmes: émeutes de subsistances et délits forestiers ${ }^{84}$. empoisonnement, vol domestique, crime passionnel. Les représentations diffusées par la presse populaire construisent cependant une image conventionnelle de la femme criminelle dont se détachent quelques figures 'dénaturées' : l'épouse empoisonneuse, la tueuse d'enfants ou la parricide, puis l'avorteuse dans le contexte nataliste des guerres mondiales. Les transformations du traitement reflètent l'ambiguïté de leur position juridique partiellement irresponsable, elles sont fréquemment taxées de folie et enfermées dans les institutions asilaires ou les couvents; l'indulgence des juges et des jurés s'inscrit dans les conceptions culturelles de la famille et l'hypocrisie du double standard moral; la place dans les prisons reste marginale et marginalisée dans l'historiographie. Seul le traitement réservé aux mineures délinquantes par les réformes de la justice a fait l'objet de recherches ${ }^{85}$. Pas étonnant que ce milieu soit mieux connu, la présence des femmes (religieuses, dames patronnesses, puis assistantes sociales) y est plus grande parmi le personnel de prise en charge. Car cette minorisation apparente des femmes par le système judiciaire s'inscrit dans une ségrégation des rôles qui n'est mise à mal que par l'arrivée au $\mathrm{XX}^{\mathrm{e}}$ siècle des femmes au barreau, puis après la seconde guerre dans la magistrature. La féminisation des professions de justice soulignées par les sociologues entraîne-t-elle pour autant une "pénalisation" croissante des femmes dans le système pénal? La question reste ouverte.

Si la question du genre traverse le droit et la justice, les productions du droit permettent de rendre la parole aux femmes et de lever le voile sur les tabous du genre. C'était la perspective d'Ann-Louise Shapiro dans son travail sur la criminalité féminine dont la construction s'inscrivait dans la dynamique de la société parisienne fin-desiècle $^{86}$. C'est résolument la perspective de la monumentale thèse d'Anne-Marie Sohn qui cherche a reconstruire la vie sexuelle des Français à travers le prisme des dossiers 
judiciaires, cours d'assises ou tribunaux correctionnels ${ }^{87}$. L'entreprise est monumentale et la plongée dans les représentations de la vie sexuelle ordinaire très convaincante en raison de l'accumulation des récits tirés des dossiers judiciaires. L'archive judiciaire permet une plongée dans les alcôves, à condition d'être lucide sur les biais du récit : réinterprétation par les acteurs en fonction des attentes supposées des magistrats, épuration des propos réels dans le langage du greffier, distorsion sociale qui laisse les bourgeois en dehors du champ de l'instruction. D'autres sources : «journaux intimes, mémoires, travaux des observateurs sociaux (....) livrent à l'occasion des instantanés précieux $»^{88}$. Principal résultat, une remise en perspective historique de la révolution sexuelle, inventée en 1968, mais aboutissement du "cheminement souterrain d'une émancipation amorcée dès 1870-1880 ${ }^{89}$. Les étapes de ce cheminement sont précisées et datées : "rupture éthique sous la III République », qui permet au sexe de se dire (sur le plan verbal). Audace plus grande des gestes, qui se généralise dans l'entre-deux-guerres par la " percée du baiser sur la bouche, de la nudité et des caresses buccales $»^{90}$, multiplication des liaisons prénuptiales et tolérance plus grande pour les passades et les adultères. Cette liberté nouvelle reste limitée, et va de pair avec une «dénonciation de plus en plus véhémente des violences sexuelles, et d'abord de celles exercées contre les enfants ". L'auteur remarque que cette émancipation des corps et des esprits se fait à l'insu des observateurs et à l'encontre des discours dominants. Elle est surtout le fait des femmes des classes populaires puis des bourgeoises. La déchristianisation et le recul des grossesses non désirées permettent aux femmes d'accepter une sexualité extraconjugale, et fait de l'amour le principal ciment du couple. Certes le double standard moral subsiste et la femme n'est pas l'égale de l'homme dans la quête de relations et dans certains milieux, la honte et l'honneur conduisent encore à l'ostracisme. Dans cette gigantesque enquête, on peut regretter cependant que le choix d'effectuer les dépouillements dans diverses régions de France n'ait pas permis de reconstruire des identités régionales sur base des 7000 dossiers analysés. À postuler dans la France de 1850 à 1950 une "nationalité des comportements ", tout entière orientée par le clivage dominant du " genre ", l'impression demeure d'aboutir à une image déterritorialisée de la vie sexuelle et d'oublier que dans le domaine sexuel comme dans d'autres, l'identité française s'est construite sur des cultures régionales et sociales.

\section{Logiques de la régulation : l'État, ses polices et ses prisons}

Si l'on aborde l'autre aspect de la déviance, les réponses sociales qu'elle suscite, l'emprise de l'État sur la régulation sociale apparaît un phénomène majeur de la période 1800-2005.

Au delà des interprétations sur le régime napoléonien (continuité ou rupture envers la révolution), force est de constater que ce dernier, à côté de l'armée, a doté l'État moderne de deux agents civils de renseignement de l'exécutif : le procureur et le préfet.

Des travaux récents mettent en lumière leur rôle important, surtout en matière politique au XIXe siècle. Le maintien de l'ordre, la gestion des crises et le contrôle de la police étaient au centre des demandes du pouvoir envers les préfets ${ }^{91}$. Quant aux procureurs généraux, "véritables officiers de renseignements généraux", ils se devaient d'être attentifs à la situation politique mais aussi morale, ce qui comprenait l'ensemble des activités des justiciables du ressort ${ }^{92}$. Ces derniers étaient mieux armés pour obtenir 
de l'information grâce aux relais locaux parmi les populations qu'étaient les juges de paix alors que les préfets dépendent des rares commissaires de police. Concurrence et rivalité entre ces différentes filières d'information, notamment pour le contrôle de la police administrative et judiciaire offrent à l'historien des sources inestimables. Les sources préfectorales, judiciaires et policières sont apparues comme déterminantes pour comprendre les rapports entre l'État et ses populations.

\section{L'État et ses polices}

Comme pour l'époque moderne, l'histoire de la police, et à travers elle, des mutations du contrôle social dans les sociétés contemporaines est un des chantiers les plus prometteurs de l'histoire politique et sociale ${ }^{93}$.

53 La diversité des polices est centrale dans le processus depuis Napoléon ${ }^{94}$. Qui pense police pense d'abord à la police parisienne, sa préfecture, ses sommiers et son commissaire Maigret. En 1994, René Lévy précisait que jusqu'alors l'essentiel de l'effort de recherche « a porté sur la période 1848-1914 » dans le fil du travail modèle de Jean-Marc Berlière consacré à la III République. Remarquant que la « période 1875-1914 est celle où apparaissent les traits distinctifs de la police actuelle: spécialisation des services, définition des missions, professionnalisation, amorce d'unification technique, emploi de la criminalistique $"^{95}$.

Dans son rapport à l'État, la police de la III ${ }^{e}$ République ne résiste pas à l'exercice du pouvoir et ne met pas fin à la division des polices. L'innovation majeure est la création d'une véritable police judiciaire nationale : les célèbres «brigades du Tigre » créées par Clemenceau en 1907. Dans le domaine du maintien de l'ordre, ces années sont caractérisées par un déclin de la violence répressive, manifeste à Paris sous l'action du préfet Lépine.

\section{A. Police en capitale}

Comme pour d'autres capitales continentales, la police parisienne est caractérisée par la concentration des trois fonctions policières principales: police d'ordre, police criminelle, police de sécurité. Le maintien de l'ordre est intimement lié aux troubles sociaux, et de manière plus subtile dans les régimes fragiles à la police politique. La police criminelle est liée aux mutations de la lutte contre la criminalité. Quant à la police de sécurité, elle présente différents aspects : contrôle des espaces locaux, des trafics (commerce, circulation routière, des activités nocturnes, des populations mouvantes, de la prostitution). À Paris, à la fin du XIXe siècle, ces trois fonctions s'exercent face à trois menaces qui s'entretiennent elles-mêmes. La peur de la populace dépravée nourrit la peur de l'insécurité qui nourrit la peur de l'émeute ou de l'attentat anarchiste ${ }^{96}$.

56 Capitale du travail, le Paris de la III ${ }^{e}$ République, est aussi capitale du plaisir depuis l'Ancien Régime ${ }^{97}$. Un des laboratoires policiers de la modernité, est la police des mœurs étudiée par Jean-Marc Berlière. Ce dernier «s'intéresse surtout à la façon dont les policiers voyaient et traitaient la prostitution » et à la place qu'occupait cette police dans le fonctionnement général de l'institution ${ }^{98}$. 


\section{B. Polices urbaines}

57 Mais Paris n'est pas la France, et la préfecture de police n'est pas le seul modèle policier. Dans sa thèse, Marie Vogel «met l'accent sur l'autonomie des villes dans le développement des polices urbaines avant l'étatisation de 1914 et étudie les rapports entre administration municipale et autorités centrales ${ }^{99}$. Son travail remet en cause l'image négative des polices municipales et rappelle que cette image fut souvent véhiculée par les partisans de la centralisation étatique. Sur la base du cas de Grenoble, l'auteur étudie la transformation vers la fin du XIX ${ }^{\mathrm{e}}$ siècle d'une police généraliste - au sens du terme d'Ancien Régime - pourchassant les chiens errants, les immondices illicites et les querelles de voisinage, à une police de plus en plus obsédée par l'activité judiciaire comme mesure de son effectivité. Il y a sans doute là l'indice d'une transformation de la police en raison des priorités municipales (la lutte contre l'insécurité ressentie à la fin du siècle), l'indice d'une acculturation aux priorités de l'État national (une déterritorialisation des menaces), mais peut-être également la mutation des formes de recours des populations à la police, dorénavant perçue non plus comme médiateur infrajudiciaire mais comme agent de la poursuite judiciaire ${ }^{100}$.

Autre monde policier investigué, celui des détectives et agences de recherche privées. Polysémie des appellations, faible visibilité du métier, archives squelettiques, souci obsessionnel du secret peuvent laisser penser qu'il n'y a pas d'histoire possible des "privés ». La posture choisie par Dominique Kalifa est celle du détective traquant les traces avouées des "privés». Archives policières, annuaires et tracts publicitaires permettent une triple d'approche "d'histoire des professions, d'histoire des représentations, d'histoire du contrôle social ». Issus des agents d'affaires, les détectives émergent comme agents de renseignements vers 1830 et se consacrent essentiellement à la "gestion des risques familiaux et à la protection des biens ». Reconnus en 1942, ils se transformeront en agents de sécurité des privés en délaissant les secrets de famille pour l'intérêt des entreprises. L'échec relatif de la police privée c'est l'échec de sa légitimation - à l'inverse du monde anglo-saxon - dans une société considérant le privé comme une "ombre». Dominique Kalifa souligne ici combien les représentations peuvent être effectives sur le recrutement et le développement ou le déclin d'une profession. Enfin, dans ses rapports à la société, la police privée semble mue par un paradoxe. Celui d'être idéologiquement en phase avec le libéralisme, la hantise de l'État et la volonté d'assurantialisation. Ce qui amène les privés, suggère Kalifa, à remplacer les médiateurs traditionnels le notaire, le médecin, le curé, puis le policier dans les affaires d'honneur, de négociation et de gestion du secret dans une société rendue plus complexe par sa croissance économique. Or en France, la profession connut un échec relatif, que l'auteur explique par la forte centralisation administrative rendant la police privée comme «un inadmissible empiètement sur les prérogatives de l'État souverain $»^{101}$. Le libéralisme en France prit une forme étatique, il ne put laisser sa place au détective privé.

\section{Polices rurales}

Principal acteur de la pacification des campagnes, la gendarmerie était restée dans l'ombre de l'histoire. Hormis des travaux pionniers sur la maréchaussée d'Ancien Régime $^{102}$, et une histoire "professionnelle " du corps, avant les années 1990, on ne disposait guère de travaux réellement scientifiques ${ }^{103}$. Les recherches de Clive Emsley 
sur les gendarmeries européennes et le monde rural, quelques synthèses régionales ${ }^{104}$ et l'important chantier mené par Jean-Noël Luc et le Service historique de la Gendarmerie Nationale nourrissent désormais une impressionnante bibliographie. Deux numéros spéciaux de la revue d'histoire de la gendarmerie ${ }^{105}$, les actes d'un colloque sur Gendarmerie, État et société au XIXe siècle et d'un autre sur le XX $\mathrm{X}^{\mathrm{e}}$ siècle ${ }^{106}$, un Guide de recherche très détaillé107, un volume sur l'histoire des représentations des gendarmes ${ }^{108}$, des dizaines de maîtrises et de thèses en cours labourent les très riches archives laissées par les gendarmes depuis deux siècles. Impossible de déployer toutes les facettes de ces recherches. Suivant le plan du colloque de 2001, et les brillantes synthèses partielles de Jean-Noël Luc, on peut rassembler les matériaux. Des études sur la gendarmerie depuis 1796 jusqu'au $\mathrm{XX}^{\mathrm{e}}$ siècle couvrent la France métropolitaine, ses colonies et ses territoires d'Outremer, en temps de guerres, de paix ou de crises intérieures. Dans cette riche historiographie, on peut dégager quatre angles d'attaque : l'évolution des fonctions assignées au corps par l'État à travers l'analyse des règlements successifs et des réformes avortées ou réussies. L'organisation de fait, en particulier la répartition des effectifs et l'implantation territoriale des brigades. Les fonctions réelles à travers la multiplicité des pratiques, réquisitions et activités et leurs interactions avec la société contrôlée. Et enfin, le profil sociologique des gendarmes et leur insertion dans la société locale. Les premiers résultats esquissent un corps écartelé entre ses fonctions militaires, judiciaires et policières au gré des régimes, une certaine fidélité aux têtes successives de l'État sans instrumentalisation excessive par ces têtes, l'évolution à la fin du XIXe siècle vers des fonctions judiciaires plus que militaires, une implantation non seulement rurale mais périurbaine, voire urbaine, les rapports contrastés avec les populations à la fois menacées et rassurées par les gendarmes, leur professionnalisation croissante au $\mathrm{XX}^{\mathrm{e}}$ siècle...

Quant aux rapports de la police et des populations, Pierre Miquel dans un style et une orientation de récit grand public, en dresse un tableau vivant à partir des «mains courantes » des îlotiers parisiens du temps de Lépine à celui de la Libération. La présentation d'une source impressionnante malgré ses lacunes met l'historien en appétit. "On peut considérer leur exploitation comme une contribution non négligeable à l'histoire de la police. Mais dans la mesure où Paris reste la matrice de l'histoire de France, ces séries ont sans doute beaucoup à dire sur l'évolution des mentalités dans le siècle, et sur les crises violentes qui ont affecté la société. Ainsi peut s'intégrer au discours historique le témoignage de la police des rues $»^{109}$.

61 Hélas, en raison du choix de ne donner aucune référence précise des nombreuses informations, on ne peut s'accorder avec lui sur ces remarques, valables par ailleurs pour toute recherche même non parisienne...

\section{La prison, de la monarchie à la République}

62 Si les origines du système pénitentiaire ont été bien étudiées, notre connaissance de l'archipel carcéral au XIXe siècle reste fragmentaire ${ }^{110}$. Récemment, par la republication de ses articles consacrés aux théoriciens et aux réalités de la vie carcérale en prison, Michelle Perrot proposait un point sur l'histoire des prisons au XIX ${ }^{\mathrm{e}}$ siècle ${ }^{111}$. Elle y constate combien les années 1970, années de critique des institutions totales ont été fructueuses pour les travaux sur la prison, ce que l'on retrouve dans la postérité de Surveiller et punir ${ }^{112}$. Si la période d'émergence (1780-1870) est balisée par 
l'incontournable opus de Jacques-Guy Petit, si la prison politique est éclairée par JeanClaude Vimont ${ }^{113}$, les bagnes popularisés par les travaux de Michel Pierre et d'Odile Krakovitch $^{114}$, la prison des femmes demeure, elle, dans l'ombre ${ }^{115}$. Pour la $\mathrm{III}^{\mathrm{e}}$ République, les travaux sont moins nombreux. En 1993, le séminaire sur la prison républicaine fait le point sur les années 1870 à 1914. Pour la plupart des auteurs, la République n'a pas supprimé la prison, elle l'a intégrée ${ }^{116}$. Certes, elle s'efforce d'y envoyer le moins de condamnés possible. Néanmoins conclut Robert Badinter, «La prison républicaine ne pouvait être qu'à l'image de la société de l'époque: dure aux pauvres, sévère dans ses pratiques disciplinaires", dont les détenus sont souvent les exclus, les marginaux des classes laborieuses ${ }^{117}$. Sans compter que le bagne accueille en pleine crise économique - 1885 - les multirécidivistes et ce jusqu'au cœur du XX $\mathrm{X}^{\mathrm{e}}$ siècle $^{118}$.

Autre catégorie soumise à l'enfermement, les enfants. L'archipel des institutions pour mineurs, symbolisé par les colonies pénitentiaires de Mettray ou Belle-Île-en-Mer fait l'objet de recherches récentes brisant les chronologies politiques des régimes ou des guerres mondiales ${ }^{19}$. Le modèle philanthropique des années 1830-1850 connaît une longue agonie jusqu'en $1937^{120}$. Une autre approche, originale est la relecture des rapports privilégiés entre religion et enfermements $d u \mathrm{XVII}^{\mathrm{e}}$ au $\mathrm{XX}^{\mathrm{e}}$ siècle. On y retrouve des travaux inspirés par la critique de Surveiller et Punir. Qu'il s'agisse d'enfermer pour dresser, soigner ou redresser avec des études sur les institutions correctionnelles pour enfants, les asiles d'aliénés, l'aumônier et le médecin en prison, reposent pour la France le lien entre "univers religieux, éthique libérale et économie de l'enfermement ", bien décrit par Jean-Marie Fecteau pour le Québec ${ }^{121}$. Nombre d'études pour nuancer ou critiquer cette impression d'immobilisme d'un siècle d'emprisonnement pénal.

Le XIX ${ }^{\mathrm{e}}$ siècle apparaît donc comme le siècle de la formation d'une vision «nationale " du crime, de l'étatisation des institutions judiciaires et de l'»acculturation » de ces deux « constructions " par les populations. Inscription dès 1800 des agences dans des hiérarchies administratives strictes, création de représentations partagées du crime, diffusion dans de plus larges couches de la population des figures du crime, de son contrôle et de sa répression : le monstre, l'apache, le gendarme, le journaliste, le préfet, le procureur, le juge, le forçat et le bagnard.

\section{Les modernisations du XXe siècle : entre civilisation et brutalisation}

On ne peut faire l'impasse dans l'histoire du $\mathrm{XX}^{\mathrm{e}}$ siècle sur les mutations répressives engendrées par les guerres civiles, mondiales, coloniales ou ethniques... Ce phénomène massif, accentué par la prise de parole des victimes, parfois concurrentielle contribue à interposer entre l'historien du contemporain et la société sous examen, des couches mémorielles des plus compactes. Tout travail historien devient un travail de déconstruction des mémoires et de reconstruction d'un ensemble de décisions, de pratiques et de refus. Une histoire sociale, doublée d'une histoire culturelle, et parfois également d'une instrumentalisation médiatique voire judiciaire.

La concurrence se manifeste dans le choix des thèses qui oriente les carrières. Il est de bon ton pour l'historien(n)e de choisir un sujet polémique, douloureux. Réviser un sujet émotionnel, obtenir des dérogations, être le premier sur un terrain controversé 
garantit un écho médiatique fort, et sans doute un poste ou une promotion rapide... Dans ce contexte, la dérive est à craindre. Un examen même sommaire des grands travaux actuels montre que la qualité des recherches n'en est pas affectée. La plupart des historiens veulent éviter «l'histoire en noms propres» tout en limitant l'autocensure et pratiquant la révision historiographique. Replacer les événements dans un schéma explicatif complexe oblige les chercheurs à se situer par rapport à leur sujet et à retracer le parcours de leur recherche.

Du point de vue thématique, deux approches nous paraissent diriger le développement du champ.

68 - Celle qui s'intéresse aux grandes administrations du contrôle social et de leur lien avec l'État d'une part, le débat citoyen de l'autre. Ainsi en est-il de l'institution policière, de la magistrature, ou du monde pénitentiaire.

69 - Celle qui étudie des pratiques concrètes de la violence étatique : celle du viol pendant la première guerre, de la tonte à la Libération, celle de la torture durant la guerre d'Algérie, celle de l'internement administratif autour des deux guerres mondiales.

\section{Le contrôle social entre État, administrations et population}

\section{A. Polices, politiques et population}

$70 \mathrm{Au} \mathrm{XX}$ siècle, les périodes de crise ont focalisé l'attention sur les liens entre politique, police et populations. En conséquence, l'entre-deux-guerres « reste un terrain vierge » tandis que Vichy constitue le point de cristallisation des rapports ambigus entre politique et police. Les rapports sont analysés sous le triple angle de la fuite en avant " souverainiste » de Vichy face aux exigences allemandes, de la participation policière à la lutte contre la résistance, et à la politique nazie de déportation et de la récupération de l'expertise de la police politique dans la lutte anti-communiste à la Libération. À travers l'étude de l'épuration policière, c'est le tableau d'une police sous occupation qui transparaît... " 'Collabos', 'fascistes ", attentistes, résistants... les policiers? La réalité est un peu moins simpliste et la position des policiers souvent intenable... $»^{122}$. Formés sous la IIIe République en "professionnels, obéissants et disciplinés, esclaves de la loi, soumis au gouvernement, indifférents aux alternances politiques (....) Ils eurent des attitudes qu'on avait toujours attendu d'eux »123. Étonnés d'avoir participé à cette zone grise privilégiée durant l'Occupation, ces policiers abdiquèrent leur «républicanisme» au profit d'un bureaucratisme non bridé par les principes démocratiques. À la Libération, l'épuration qui visa surtout à retourner d'un bloc la police au service du nouveau gouvernement, resta incomprise de la plupart des agents, et coûta à la IVe République la fidélité de sa police lors des troubles de 1958.

\section{B. Justice, magistrature, politique}

71 Donner à l'appareil judiciaire et ses pratiques le statut d'une administration et d'une institution susceptible d'analyse sociopolitique est un courant récent et en plein développement dans l'historiographie française. Il est vrai que, pour ne s'en tenir qu'au $\mathrm{XX}^{\mathrm{e}}$ siècle, les traumatismes ayant affecté les relations entre l'État, sa justice et ses citoyens n'étaient pas minces: de l'affaire Dreyfus à la guerre d'Algérie, les interventions du politique dans l'exercice de la justice foisonnent. Cent ans après l'affaire Dreyfus, «La densité des interrogations découlant de la relation entre justice et 
politique, la possibilité de construire un objet historique où la magistrature serait analysée comme acteur de la République, la nécessité, enfin, de prendre en compte le questionnement de la société sur une «République des juges » dénoncée ou applaudie » rendait nécessaire l'étude des rapports entre "mécanismes judiciaires, enjeux politiques et valeurs républicaines" c'est-à-dire entre "les juges, les gouvernants et la cité »"124. Il s'agit tout à la fois de contribuer à éclairer les rapports de la justice avec le pouvoir politique, interventions du politique dans l'activité judiciaire ou moyens qu'a l'État comme administration de ne pas tenir compte des décisions de justice. Une pratique politique au cœur de ce rapport est l'amnistie, aujourd'hui décriée mais qui aura accompagné un long siècle d'histoire française, est jalonnée de périodes critiques pour les rapports entre politique et justice: la Commune, la Première Guerre mondiale, Vichy, la Libération et l'Algérie ${ }^{125}$.

72 La thèse d'Alain Bancaud nous confronte à la relégitimation de la magistrature des années 1940 à la Libération. Une magistrature cultivant « des vertus moyennes », peu différente de la description du parquet royal à la fin de l'Ancien Régime ${ }^{126}$, naviguant entre les régimes et les justifications contradictoires. Elle inscrit la recomposition de la libération dans l'histoire longue des épurations judiciaires, caractéristiques des tensions entre politique et judiciaire ${ }^{127}$.

L'attitude des magistrats pendant la guerre d'Algérie fait l'objet de la thèse de Sylvie Thénault. Si l'armée se fixe comme objectifs de "prendre en main la population», elle n'est cependant pas à l'origine de l'intervention de la justice dans la guerre d'Algérie. C'est le pouvoir politique qui lui assigne la mission principale : servir d' » instrument de lutte contre les indépendantistes algériens ». "L'État de droit se dilue alors dans la guerre et les magistrats (...) sont confrontés à la réalité de la torture qui souille l'enquête préliminaire, tandis que leurs décisions subissent l'affront d'une révision par l'armée qui prend pour habitude d'interner les bénéficiaires d'un non-lieu, d'un acquittement, d'un sursis ou d'une libération de prison ${ }^{128}$. Grâce aux archives militaires, des ministères de la Justice et de l'Intérieur, des papiers d'avocats et quelques entretiens, S.Thénault estime avoir pu replacer les dénonciations militantes, les réflexions sociologiques et juridiques dans "la très forte dimension humaine de ce très proche passé $»^{129}$. Le portrait de la magistrature qui en résulte est celui d'une "grande muette». De par son recrutement aisé, et faute de formation professionnelle et d'expression syndicale, la magistrature des années cinquante apparait selon la formule de Casamayor davantage "une machine judiciaire faite de textes et de juges qui les appliquent, qu'un pouvoir judiciaire indépendant, tenant la balance égale entre le Pouvoir et le citoyen ». Ce n'est que sur leurs qualités individuelles que certains magistrats s'opposèrent à la logique répressive. Concluant comme pour les hauts magistrats (Bancaud) ou les policiers de l'Occupation (Berlière, Chabru), «dans leur immense majorité, par conséquent, les magistrats ont joué le rôle qui leur était demandé, avec plus ou moins de zèle et d'empressement selon les cas $»^{130}$.

\section{L'archipel de l'internement}

Le $\mathrm{XX}^{\mathrm{e}}$ siècle a été le siècle des camps ${ }^{131}$. L'extraordinaire développement de l'internement constitue un troisième domaine de l'exception liée à la guerre. S'interdisant de comparer les camps de "concentration" français de la première guerre avec les camps d'internement de Vichy et les camps d'extermination nazis, JeanClaude Farcy fait remarquer que la finalité des premiers "est essentiellement militaire ». Regrouper les mobilisables austro-hongrois y compris les Alsaciens « libérés ", retenir 
les otages et les notables, évacuer les indésirables (prostituées malades, repris de justice) qui sont qualifiés de "suspects nationaux ». Par ces effets administratifs : internement, fichage et contrôle, cette première expérience "concentrationnaire " s'inscrit dans l'histoire de la répression en temps de guerre ${ }^{132}$.

Ce sont encore les « indésirables étrangers » qui motivèrent la III ${ }^{e}$ République finissante à rouvrir des camps pour concentrer les réfugiés de la guerre civile espagnole. Ceux-ci furent utilisés sous l'Occupation par l'État français, puis à la Libération par la République renaissante. Denis Peschanski repère entre 1938 et 1946 quatre «logiques dominantes (donc non exclusives) à l'œuvre » : dans les derniers temps de la III République, c'est celle de l'exception qui prime; avec l'instauration du régime de Vichy, l'internement s'intègre dans une logique d'exclusion, cœur du nouvel État; à partir de 1942 se surimpose et s'impose la mise en œuvre de la Solution finale en France, voulue par les Allemands; avec la Libération, l'internement s'inscrit de nouveau dans une politique d'exception ${ }^{133}$. Ces quatre logiques de la volonté du pouvoir sont nuancées par trois cas de figure. Soit l'internement répond à un projet idéologique (communistes, droits communs, Juifs étrangers, nomades...). Soit il s'agit de prendre des mesures d'exception dans un contexte de guerre (ressortissants ennemis ou collaborateurs présumés). Soit il s'agit d'une réponse à une contrainte extérieure (Peschanski cite le cas des Juifs sarrois ou des Juifs déportés de France). Enfin, l'internement de populations entières apparaît comme un instrument administratif que l'État utilisa, que les administrations pratiquèrent et dont l'opinion ne s'émut guère pendant les années noires ${ }^{134}$.

\section{Violences de guerres et contentieux ordinaires}

Privilégiant les périodes d'exception, la plupart des recherches d'histoire du premier $\mathrm{XX}^{\mathrm{e}}$ siècle occultent les caractéristiques de l'ordre et du désordre au quotidien. Pour le $\mathrm{XX}^{\mathrm{e}}$ siècle, en raison des restrictions de consultation d'archives, l'historiographie française n'a pas développé de grandes synthèses régionales comparables aux travaux sur les sociétés rurales du XIX siècle. Monde rural, bassins industriels ou sociétés urbaines du XXe siècle dans leur rapport au crime et à la justice sont donc mal connus et leur évolution malaisée à situer dans un vaste XIX siècle qui court de 1750 à 1914.

Les guerres et en particulier la seconde et l'Occupation, ont monopolisé l'intérêt, en tentant de replacer la spécificité de la période dans une séquence plus large: des années 1930 aux années 1950. Il n'empêche, seul le travail sur les statistiques de Compte général permet de placer un décor derrière la scène des violences d'exception. Car «dans son fonctionnement 'ordinaire', la justice pénale est alors (...) marquée par des traits exceptionnels $»^{135}$.

On y voit apparaître le marché noir, tandis que le nombre de condamnés pour vol fait plus que tripler ${ }^{136}$. Ces deux "types d'infractions motivent l'essentiel de l'augmentation considérable des condamnations à l'emprisonnement ferme entre 1938 et $1942 »^{137}$. Ceci ne doit pas masquer un second effet de l'Occupation, l'accélération d'une évolution commencée auparavant et consolidée par après.

Parmi ces mouvements, l'augmentation des condamnations pour avortement. Le code de 1791 ne prévoyait aucune peine contre les femmes avortées tandis que les avorteurs étaient poursuivis de vingt ans de fer. Le code de 1810 en revient à la répression de l'avortée et des avorteurs ${ }^{138}$. Faute d'études précises, il est difficile de mesurer les 
ruptures des $\mathrm{XIX}^{\mathrm{e}}$ et $\mathrm{XX}^{\mathrm{e}}$ siècles. Il semble que le $\mathrm{XIX}^{\mathrm{e}}$ siècle soit celui de la "généralisation de l'avortement" et de son extension massive à toutes les couches sociales. Sans doute, l'avortement remplace-t-il progressivement l'infanticide comme moyen d'éviter les enfants non désirés. Mais c'est entre 1920 et 1945, en conséquence directe de la guerre que la répression fut la plus visible et la plus vivace. Une répression affirmée dans un contexte de crainte dépopulationniste et récupérée par un régime totalitaire dans le cadre d'une vision nationaliste de la famille.

\section{A. Ordre moral et politique de la famille}

judiciaire. "Avec les viols et les enfants du viol, on touche aux enjeux idéologiques de la Première Guerre mondiale». Examinant le problème posé à la morale politique par ces comportements, à la lueur de la "culture de guerre» Stéphane Audouin-Rouzeau remarque que "c'est le sang allemand, par nature corrompu et corrupteur qui, aux yeux d'un certain nombre d'intervenants dont on a examiné les positions dans ce livre, rend inassimilables les enfants du viol, et excuse, justifie, voire légitime, non seulement l'avortement, mais parfois l'infanticide ${ }^{139}$. Dans la ligne d'une histoire culturelle de la violence de guerre, Jean-Yves Le Naour aborde, essentiellement sur base des discours, la vie sexuelle durant la Grande guerre. Mis à part le cas des amours avec l'ennemi en zone occupée ${ }^{140}$, l'autonomie apportée par la guerre aux jeunes filles, l'attention portée aux comportements des femmes de soldats, l'hypocrisie de la prostitution à usage des militaires dessinent un tableau tourmenté des rapports de sexes.

Pour la seconde guerre, les recherches se sont concentrées sur les politiques juridiques et judiciaires du régime de Vichy. Prenant à rebours la confusion entre " ordre moral » et politique de Vichy, Marc Boninchi analyse finement la genèse d'une série de législations pénales touchant les infractions à l'ordre moral : adultère, homosexualité, prostitution, alcoolisme et avortement ${ }^{141}$.

C'est par l'entrée d'histoire de la sexualité que Cyril Olivier aborde les politiques de Vichy dans le domaine des mêmes «sexualités féminines " ${ }^{142}$. La complémentarité des deux enquêtes est manifeste: en historien du droit, le premier se focalise sur les papiers des bureaux chargés de préparer les lois, le second, en historien du genre et de la société, interroge les archives des tribunaux correctionnels. Ni l'un, ni l'autre n'oublient cependant l'autre facette de la dialectique entre création normative et applications par les juridictions, en raisonnant notamment sur des dossiers judiciaires et les statistiques du compte général.

En soulignant la faible opérationnalité des projets du ministère de la Famille, le travail de Boninchi renforce le constat de Cyril Olivier «s'agissant du gouvernement de Vichy, les archives judiciaires renseignent bien davantage sur l'état de la société et des représentations visà-vis des sexualités, que sur le pouvoir en lui-même, qui n'est finalement pas très écouté, sinon entendu par les magistrats du siège $»^{143}$.

Tous deux relativisent donc le durcissement d'un prétendu "ordre moral» des partisans de la Révolution nationale en le replaçant dans une scansion plus longue (1920-1960) où pratiques scientifiques (l'eugénisme), angoisses morales (la dénatalité) et peurs sociales (le déclin national) trouvent dans un régime autoritaire un instrument d'une attention renouvelée sur la régulation des sexualités hors mariage monogame. En même temps, ils soulignent les résistances des fonctionnaires comme des magistrats à

Crime, Histoire \& Sociétés / Crime, History \& Societies, Vol. 10, n² | 2006 
verser dans une inquisition sociale des populations au nom d'une vision catastrophiste de la cohésion nationale. Enfin, ils confirment la spécificité des périodes de guerres et d'occupations qui brouillent et recomposent les identités de genre ${ }^{144}$.

Entrées en guerre et sorties d'occupation sont marquées par une crise de l'autorité légitime et une résurgence des châtiments "collectifs » ${ }^{145}$. C'est ici que prend toute sa place un concept social complexe, étendu désormais à l'ensemble d'une population : l'épuration.

\section{B. Fusillades, violences populaires et épurations}

Avec les fusillés de 1917, c'est le débat sur la justice militaire, ces liens avec le politique et la raison d'État qui rebondit. Reprenant le dossier solidement établi par Pedroncini, Nicolas Offenstadt, rappelle que dès l'entre-deux-guerres, le retour en grâce des fusillés était à l'ordre du jour, mais qu'il fallut la prise de confiance victimaire généralisée pour leur assigner une place de «martyrs de la grande guerre ${ }^{146}$. Puis André Bach reprend magistralement la question, en mettant en lumière les fusillades de 1914-1915, bien plus mortifères que celles de 1917. Confirmant une tendance dans les armées alliées, il note que sur un total d'environ 600 fusillés, près de 430 soldats français ont été passés par les armes entre septembre 1914 et décembre 1915. Face à une justice militaire aléatoire, il met en évidence le contrôle parlementaire qui aboutira par la proposition de loi du 24 avril 1916 à endiguer la vague répressive consécutive aux mutineries de 1917.

87 Si la Libération de 1944 fut l'occasion d'une politique d'internement des collaborateurs présumés, elle fut aussi marquée, dans un contexte de déroute des pouvoirs institués de manifestations de violence "populaire ». La tonte des femmes magistralement étudiée par Fabrice Virgili en est un exemple. L'image de la «tondue » brouille le phénomène réel. «Or la diversité des 'tontes' interdit toute explication univoque. Ces tontes concernent les sphères publique et privée, les rapports entre les sexes et les relations entre occupants et occupés: elles sont une violence physique et symbolique, un acte punitif avec une dimension combattante... elles sont un marqueur des contradictions qui traversent une société française en plein bouleversement $»^{147}$.

88 Virgili souligne le lien entre épuration extra-judiciaire et épuration légale ${ }^{148}$. L'imbrication entre les deux formes d'épuration est réelle et leur frontière poreuse. Acte social, la tonte permet d'étudier l'articulation entre les vécus individuels et collectifs de la guerre. L'exercice de la violence constitue un moment de communion nationale. Le caractère reconstructeur de l'identité, du territoire et du pouvoir après le traumatisme de juin 1940 et les années noires de l'Occupation justifie le titre de «France virile » sur la couverture de cette recherche sur les tontes de la Libération. À partir de cet objet de la tonte, comme Audoin-Rouzeau l'avait abordé pour le viol, un éclairage global d'une société en crise se dessine, articulant destins individuels et comportements collectifs, rupture avec le passé et reconstruction de l'avenir. Tonte de

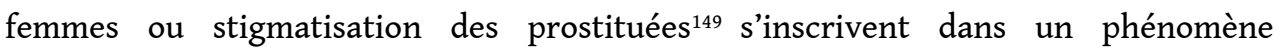
chronologique plus large (1945-1960), varié, polysémique des réactions sociales à la guerre ou à l'occupation, telles qu'abordées une fois de plus de manière neuve, grâce à l'apport des sources judiciaires.

89 L'histoire de l'épuration après la seconde guerre est proche de celle d'un fait social global. Susceptible d'une approche par en haut, en termes de politique répressive à 
l'instar du cas belge ${ }^{150}$, elle s'enrichit d'études locales et micro-historiques tant le phénomène épuratoire relève d'une "construction historique des formes complexes de relations entre l'individu, le groupe et l'événement $»^{151}$. Croisant des niveaux d'échelle différents, articulant des demandes sociales avec des pratiques juridiques, variant dans le temps et l'espace, l'enquête nationale ${ }^{152}$ s'enrichit d'études régionales ${ }^{153}$. Reste à l'inscrire dans une histoire comparée des sorties de guerre européennes ${ }^{154}$.

\section{Justice et crime dans les colonies}

Le retour des politiques répressives coloniales au premier plan du débat historiographique est sans conteste lié au débat sur la guerre d'Algérie. Les travaux récents de Sylvie Thénault sur les magistrats et de Raphaëlle Branche sur la torture et l'armée s'inscrivent dans les orientations décrites précédemment. Pratiques concrètes, justifications juridiques, influences politiques. Le problème de la définition de la torture est bien évoqué par Raphaëlle Branche. "Les définitions de la torture qui la lient à une fin extérieure, telle que l'obtention de renseignements, portent en elles l'euphémisation de ces violences, notamment celles qui sont accomplies sans but repérable. Au contraire, il faut resserrer la définition sur trois éléments fondamentaux qui ignorent cette fin apparente: la souffrance infligée, l'intention de celui qui l'inflige, et enfin la volonté d'ôter à l'autre sa capacité de penser, qui est au coeur de cette intention". Mais continue l'auteur "ce recentrage ne signifie aucunement se limiter au bourreau et à sa victime (....) ». La torture est le cœur d'un système répressif, "conçu comme nécessaire pour gagner cette guerre-là » ${ }^{155}$. Comme la tonte à la Libération, la torture permet de saisir la guerre et s'inscrit « dans une histoire plus vaste de la violence de guerre ». Entre 1957 et 1960, institutionnalisée par l'armée, la torture servait à la fois le renseignement et la terreur. L'usage de l'électricité «peut même être considéré comme une marque de la civilisation française » face aux Algériens « qui faisaient souffrir en répandant le sang $\aleph^{156}$. Elle introduisait une distanciation, une technicisation masquant la souffrance. Rappelant le pouvoir du colonisateur, la torture n'était cependant pas dicible par l'État qui se voulait démocratique. L'auteur termine son ouvrage par une relecture mémorielle de la difficile émergence du débat sur les responsabilités dans les "opérations de maintien de l'ordre en Afrique du Nord, devenues en 1999, la "guerre d'Algérie » ».

91 La levée des tabous sur la guerre d'Algérie, ne doit pas masquer que le monde colonial reste largement en friche. Deux approches récentes sur la prison et la prostitution concernent essentiellement l'Afrique. Le double volume collectif publié par Florence Bernauld sur le contrôle social et la prison en Afrique noire et la thèse de Christelle Taraud sur le prostitution dans le Maghreb français posent le jalon de recherches à venir. «La transplantation de la prison en Afrique noire par les pouvoirs coloniaux aboutit à un rapport à l'enfermement très différent de la colonie. Privée de son volet réformateur, la prison n'est qu'un des systèmes de contrainte par corps et fonctionne comme un appendice de la mobilisation du travail des colonisés. L'organisation de la prostitution en Afrique du Nord via l'implantation d'un système répressif (...) propose une radiographie saisissante de la situation coloniale dans une combinaison insolite et explosive de racisme, de capitalisme et de moralisme ${ }^{157}$. Consciente de la surreprésentation de la vision coloniale dans la documentation écrite, l'auteur dépasse une analyse du réglementarisme colonial, en replaçant les prostituées dans leur marginalité face au contexte urbain avant de se livrer à une fine analyse des rencontres culturelles jusqu'aux guerres coloniales et aux accusations de collaboration sexuelle en période de décolonisation. De manière 
similaire, la redécouverte des contributions coloniales aux guerres mondiales enrichit le champ de la domination et de la rencontre interculturelle. Ainsi les rapports sociaux de la colonisation s'inversent dans un épisode médiatique, comme la "honte noire " des troupes coloniales française dans la Rhénanie occupée des années $1920^{158}$. Les fantasmes attisés par les résistants allemands à l'occupation envers les troupes coloniales françaises s'inscrivent aussi dans le contexte d'une guerre de propagande entre pays colonisateurs de l'Afrique noire.

\section{Historiens, meurtres de masse et justices internationales : vers une histoire globale du crime?}

Un nouveau chantier s'est ouvert avec l'imprescriptibilité des crimes contre l'humanité. Dans l'avalanche de travaux consacrés aux «meurtres de masse » et à la justice transnationale, relevons la remarquable tentative de Jacques Sémelin de cerner de manière comparée et pluridisciplinaire les mécanismes des génocides ${ }^{159}$. En conséquence, l'historien s'est trouvé confronté à une demande judiciaire et sociale d'intervention dans le processus judiciaire. L'intervention des historiens dans le prétoire, comme l'intervention de la justice, voire du politique dans la définition du travail historique ont suscité des réflexions passionnées sur le " devoir de mémoire », le rôle du témoin et de l'historien, la vérité historienne et judiciaire.

93 À son tour, l'historien rejoint les figures du crime et de la justice qu'il étudie : témoins, experts, juges... et criminels ${ }^{160}$.

\section{Conclusions générales}

«Si la justice d'aujourd'hui est forte de son insertion sociale, comment ne pas souhaiter que son histoire soit étudiée et analysée dans le cadre d'une approche globale, attentive à l'évolution lente des structures et des institutions comme au jeu des acteurs, soit celui d'une histoire sociale et politique renouvelée ? ${ }^{161}$. Le programme appelé de ses vœux par Jean-Claude Farcy paraît en partie réalisé.

L'ambiguïté du statut de l'histoire dans la construction de l'identité française conditionne trois caractéristiques de l'historiographie sur la période contemporaine.

L'internationalisation de l'historiographie française reste modeste mais se traduit par une prise en compte croissante des travaux en d'autres langues dans l'historiographie générale. Certaines périodes, comme la Révolution française, les guerres mondiales, la décolonisation sont plus propices à cette ouverture, malgré un certain décalage lié aux processus de traduction ${ }^{162}$.

97 Le comparatisme demeure limité. Quelque soit le niveau d'échelle (un département, une région, l'hexagone) la société française est largement perçue comme un monde autonome, rarement inscrit dans une société plus vaste (occidentale, européenne ou mondiale). Souvent les travaux français "parlent aux Français» et s'inscrivent rarement dans une problématique occidentale ou mondiale.

Enfin, la césure entre les historiens du droit et de lettres et les spécialistes de la société française contemporaine reste préoccupante. Dans le domaine précis du crime et de la justice, l'inculture de certains experts pérorant sur l'insécurité contemporaine 
témoigne que la finesse des apports d'histoire de la criminalité est loin d'être intégrée à la connaissance des sociétés d'aujourd'hui.

Une deuxième conclusion porte sur la place de l'histoire du crime et de la justice dans l'historiographie. Pour certains auteurs, elle est un aspect limité de l'approche que leur recherche dépasse vers une histoire des sensibilités (Lapalus, Olivier). On peut se demander, si loin d'être une spécialité cloisonnée, l'histoire du crime et de la justice porte en elle un projet d'intelligibilité globale d'une société. L'originalité de l'histoire du crime et de la justice n'est-elle pas au contraire de refuser de s'inscrire comme un champ supplémentaire d'histoire nationale dans un paysage encombré, mais une démarche de recherche où la documentation, la problématique, la méthode critique et la réflexion épistémologique rappellent que l'interprétation des évolutions du comportement humain est à la fois locale, institutionnelle, et universelle.

La remarque de Jean-Claude Farcy invite à également renouer avec le comparatisme dans le temps long. Le caractère transpériode, propre au contexte interdisciplinaire de l'histoire du crime et de la justice n'a pas réellement fait florès. Si d'emblée les travaux d'associations comme l'IAHCCJ avaient associé médiévistes, modernistes et contemporanéistes, les lieux institutionnels où s'est épanoui ce caractère transpériode sont peu nombreux. Force est de constater le maintien du cloisonnement de l'histoire générale en périodes et l'absence de synthèses résolument transpériodes. Nombreux sont les colloques qui rassemblent des spécialistes de périodes différentes, mais de manière morcelée, tant les sources, les contextes et les techniques varient. Et la synthèse se limite en général aux parties introductives ou conclusives d'ouvrages collectifs. Il y a donc place pour un véritable comparatisme temporel.

L'historien médiéval et moderne gagne à prendre en compte le regard sur les sources porté par ses collègues du contemporain. La massivité des sources, tant policières que judiciaires ou littéraires élargit le questionnement de l'historien contemporain. Archives judiciaires, papiers de police, presse populaire et débats d'opinion, romans et caricatures, l'historien des temps contemporains est privilégié par rapport à ses collègues médiéviste ou moderniste. Cette variété documentaire combine les sources typiques d'Ancien Régime : normes et archives judiciaires avec des récits plus neufs sur le crime : discours parlementaires, écrits savants, statistiques administratives, images et photographies... Bien maîtrisée, elle permet à l'historien de sortir du dilemme d'interprétation de l'archive judiciaire, "sismographe de criminalité » ou "indice d'activité judiciaire ». Les dossiers judiciaires, reliés à un terroir précis, balisés par une bonne connaissance des échelles $\mathrm{du}$ monde judiciaire peuvent renouveler profondément l'histoire sociale comme l'histoire politique. Bien plus, il n'y a pas opposition mais superposition de compréhension dans le croisement des méthodes d'analyse et des niveaux d'échelles choisis. Avec l'avantage de l'historien des XIX et $\mathrm{XX}^{\mathrm{e}}$ siècles sur celui des temps préindustriels, de pouvoir identifier les niveaux de discours et de pratiques et de saisir la lente acculturation des uns par les autres, du local par le national, puis l'international au $\mathrm{XX}^{\mathrm{e}}$ siècle et discerner les résistances et les récupérations communautaires.

En outre, l'historien du contemporain gagne à sortir d'une certaine naïveté des interprétations en terme de "jamais vu » ou "sans précédent », par la prise en compte des travaux de ses collègues engagés plus loin dans le temps, notamment pour l'étude des situations où l'État semble s'effriter face à la société. 
Pogroms, viols collectifs, tontes publiques, tortures inavouées, détention sans légalité rappellent les critiques adressées à la société médiévale ou à la justice de l'ère classique. Confronté à la perspective d'un "retour de la barbarie », souligné par la violence de guerre, l'historien du contemporain s'interroge immédiatement sur la fin du processus de civilisation et de la contention de la violence; «expliquer pourquoi la Première Guerre mondiale a fait basculer l'Europe d'une société qui semblait en voie de pacification à une société de violences et que la violence de guerre n'a été que le paravent de la violence du siècle $»^{163}$.

Ces pratiques mettent l'accent sur l'irénisme du discours de l'État de droit, de la démocratie et des droits de l'homme et du citoyen, fondé sur le voilement des horreurs. Elles soulignent l'inaptitude des détenteurs d'une parcelle de pouvoir (policiers, juges, militaires), à gérer les pratiques répressives. D'où l'insistance sur une pédagogie qui ne diabolise pas la violence en la renvoyant à l'ordre moral mais élabore une véritable éducation à la violence de générations élevées dans l'oubli et la négation des conflits sanglants. Ici les problématiques contemporaines ont intérêt à s'inspirer des recours à l'anthropologie de la régulation des conflits de leurs devanciers et intégrer leur problème dans la longue durée du phénomène. Ceux-ci rappellent que la recrudescence de ces phénomènes est parallèle à l'affaiblissement de l'État et du lien social.

\section{Généralités (compléments voir $\mathrm{CHS}, 2006$, vol.10, $\mathrm{n}^{\circ} 1$, pp. 150-151)}

Briegel F., Porret M. (eds.), Le criminel endurci. Récidive et récidivistes du Moyen Âge au XXe siècle, Genève, Droz, 2005.

Dolan C. (ed.), Entre justice et justiciables. Les auxiliaires de la justice du Moyen Âge au XX $X^{e}$ siècle, Québec, Presses de l'Université Laval, 2005.

El Kenz D. (ed.), Le massacre, objet d'histoire, Paris, Gallimard, 2005.

Fecteau J.-M., Harvey J. (eds.), La régulation sociale entre l'acteur et l'institution. Pour une problématique historique de l'interaction. Agency and Institutions in Social Regulation. Toward an historical undestanding of their interaction, Sainte Foy, Presses de l'Université du Québec, 2005. Le crime et le pouvoir, Les rendez-vous de l'Histoire, Blois 1998, Nantes, Éditions Pleins Feux, 1999. Lemesle B. (ed.), La preuve en justice de l'Antiquité à nos jours, Rennes, Presses universitaires de Rennes, 2003.

Milliot V. (ed.), Les mémoires policiers 1750-1850. Écritures et pratiques policières du Siècle des Lumières au Second Empire, Rennes, Presses universitaires de Rennes, 2006. 
Sémelin J., Purifier et détruire. Usages politique des massacres et génocides, Paris, Seuil, 2005.

Zélis G. (ed.), L'historien dans l'espace public. L'histoire face à la mémoire, à la justice et au politique, Bruxelles, Éd. Labor, 2005.

\section{Sites internet, et revues}

www.criminocorpus.cnrs.fr

Bibliographie Justice en France 1789-2004

Archives de l'Anthropologie Criminelle (1886-1914)

Histoire de la justice

Revue d'histoire de l'enfance irrégulière

Sociétés et représentations

\section{Révolution}

Allen R., Les tribunaux criminels sous la Révolution et l'Empire 1792-1811, Rennes, Presses Universitaires de Rennes, 2005.

Berger E., Le tribunal correctionnel de Bruxelles sous le Directoire, Bruxelles, Archives générales du Royaume, 2002.

Bourguinat N., Les grains du désordre. L'État face aux violences frumentaires dans la première moitié du XIX ${ }^{e}$ siècle, Paris, Éditions de l'EHESS, 2002.

Brown H., Ending the French Revolution: Violence, Justice, Repression, from the Terror to Napoleon Charlottesville, University of Virginia Press, 2006.

Brown H., Miller J. A. (coed.), Taking Liberties : Problems of a New Order from the French Revolution to Napoleon, Manchester University Press, 2002; Palgrave, 2003.

de Mari É., Le parquet sous la Révolution. 1789-1799, in Carbasse J.M. (ed.), Histoire du parquet, Paris, PUF, 2000, pp. 221-255.

de Mari É., Quelques remarques sur l'historiographie judiciaire de la Révolution, lesÉpisodiques, Lille, 2001, pp. 23-29 (numéro spécial La Révolution).

Dupont-Bouchat M.-S., Rousseaux X. (eds.), Juges, délinquants et prisonniers dans le département de Sambre-et-Meuse (1794-1814), [Namur, 1999] (Annales de la Société archéologique de Namur, t. 72, 1998).

Gueniffey P., La politique de la Terreur. Essai sur la violence révolutionnaire, Paris, Fayard, 2000.

Humbert-Convain S., Le juge de paix et la répression des infractions douanières en Flandre et en Hollande (1794-1815) : contribution à l'histoire du système continental napoléonien, Rotterdam, Erasmus Universiteit, 1993.

Joannic-Seta F., Le bagne de Brest. Naissance d'une institution carcérale au siècle des Lumières, Rennes, Presses Universitaires de Rennes, 2000 (Histoire).

Justice et institutions françaises en Belgique (1795-1815). Traditions et innovations autour de l'annexion, Lille, L'espace juridique, 1996.

Justice et politique : la Terreur dans la Révolution française, Toulouse, Presses de l'Université des Sciences Sociales de Toulouse, 1997. 
Justice et République(s). Actes du colloque de Lille, 17-20 septembre 1992, Hellemes, Estu, 1993.

Lignereux A., Gendarmes et policiers dans la France de Napoléon. Le duel Moncey-Fouché, MaisonsAlfort, Service historique de la Gendarmerie nationale, 2002.

Martin J.-C., Violence et Révolution. Essai sur la naissance d'un mythe national, Paris, Seuil, 2006.

Maza S., Private Lives and Public Affaires : the Causes Célèbres of Prerevolutionary France, Berkeley, University of California Press, 1993. Trad. française Vies privées, affaires publiques. Les causes célèbres de la France prérévolutionnaire, Paris, Fayard, 1997.

Rappaport S., La chaîne des forçats (1792-1836), Paris, Aubier, 2006.

Rousseaux X., Dupont-Bouchat, M.-S., Vael C. (eds.), Révolutions et justice pénale en Europe (1780-1830). Modèles français et traditions nationales, Paris, L'Harmattan, 1999 (Collection Logiques Sociales).

Royer J.-P., Martinage R. (eds.), Influence du modèle judiciaire en Europe sous la Révolution et l'Empire, Lille, L'espace juridique, 1999.

Shapiro B. M., Revolutionary Justice in Paris (1789-1790), New York, Cambridge University Press, 1993.

Spierenburg P., The prison experience : prisons, disciplinary institutions and their inmates in early modern Europe, London, Rutgers University Press, 1991

\section{La France contemporaine (1800-2005)}

Allinne J.-P., Gouverner le crime. Les politiques criminelles françaises de la Révolution au XXI ${ }^{e}$ siècle 1. L'ordre des notables 1789-1920, 2 - Le temps des doutes 1920-2004, Paris, L'Harmattan, 2003-2004.

Ambroise-Rendu A.-C., Peurs privées, angoisses publiques. Un siècle de violences en France, Paris, Larousse, 2001.

Arnaud-Duc N., La discipline au quotidien. La justice correctionnelle dans la Provence aixoise au XIX siècle, Dijon, Éditions universitaires de Dijon, 1997.

Artières Ph. (ed.), Le livre des vies coupables. Autobiographies de criminels (1896-1909), Paris, Albin Michel, 1999.

Aubusson de Cavarlay B., Huré M.-S., Pottier M.-L., La justice pénale en France. Résultats statistiques (1934-1954), Paris, Institut d'histoire du temps présent, 1993 (Cahiers de l'IHTP nº 23).

Aubusson de Cavarlay B., Huré M.-S., Pottier M.-L., Yordamian S., Les abandons de poursuite avant jugement et leurs motifs de 1831 à 1932 : la base DAVIDO, séries par infractions, Guyancourt, CESDIP, 2001.

Audoin-Rouzeau S., L'enfant de l'ennemi. 1914-1918. Viol, avortement, infanticide pendant la Grande Guerre, Paris, Aubier, 1995 (Aubier historique).

Audouin-Rouzeau S., Becker A., Ingrao C., Rousso H. (eds.), La Violence de guerre 1914-1945, Bruxelles, Complexe, 2002.

Bancaud A., La haute magistrature française entre politique et sacerdoce ou le culte des vertus moyennes, Paris, LGDJ, 1993.

Bard C., Chauvaud F., Perrot M., Petit J.-G. (eds), Femmes et justice pénale XIX ${ }^{e}-X^{e}$ siècles, Rennes, Presses Universitaires de Rennes, 2002. 
Baruch, M.-O., Une poignée de misérables. L'épuration de la société française après la Seconde Guerre mondiale, Paris, Fayard, 2003.

Baruch M.-O., Duclert V. (eds.), Justice, politique, et République. De l'affaire Dreyfus à la guerre d'Algérie, Bruxelles, Complexe, 2002.

Bergère M., Une société en épuration. Épuration vécue et perçue en Maine-et-Loire. De la Libération au début des années 50, Rennes, Presses universitaires de Rennes, 2004.

Berlière J.-M., Le Préfet Lépine. Vers la naissance de la Police moderne, Paris, Denoël, 1993.

Berlière J.-M., Chabrun L., Les policiers français sous l'Occupation d'après les archives inédites de l'épuration, Paris, Perrin, 2001.

Berlière J.-M.,Vogel M., Police, État et société en France (1930-1960) : essai bibliographique, Paris, CNRSIHTP, 1997 (Cahiers de l'IHTP).

Branche R., La torture et l'armée pendant la guerre d'Algérie, Paris, Gallimard, 2001.

Boninchi M., Vichy et l'ordre moral, Paris, PUF, 2005.

Bouchet T., Leggett M., Vigreux J., Verdo G. (eds.), L'insulte (en) politique Europe et Amérique latine du XIX ${ }^{e}$ siècle à nos jours, Dijon, Éditions universitaires de Dijon, 2005.

Bourdin Ph., Caron J.-C., Bernard M. (eds), La voix et le geste. Une approche culturelle de la violence socio-politique, Clermont-Ferrand, Presses universitaires Blaise-Pascal, 2005.

Capdevila L., Les Bretons au lendemain de l'Occupation : imaginaire et comportement d'une sortie de guerre, 1944-1945, Rennes, Presses universitaires de Rennes, 1999.

Caron J.-C., À l'école de la violence : châtiments et sévices corporels dans l'institution scolaire du XIX siècle, Paris, Aubier, 1999.

Chauvaud $\mathrm{F}$., Le juge, le tribun et le comptable. Histoire de l'organisation judiciaire entre les pouvoirs, les savoirs et les discours (1789-1930), Paris, Economica, 1995 (Anthropos Historiques).

Chauvaud F., Les criminels du Poitou au XIX ${ }^{e}$ siècle. Les monstres, les désespérés et les voleurs, La Crèche, Geste éditeur, 1999 (Pays d'histoire).

Chauvaud F., Les experts du crime. La médecine légale en France au XIXe siècle, Paris, Aubier, 2000.

Chauvaud F., Dumoulin L. (eds.), Experts et expertise judiciaire. France, $\mathrm{XIX}^{e}$ et XX $\mathrm{X}^{e}$ siècles, Rennes, PUR, Collection Histoire, 2003.

Chauvaud F., Mayaud J.-L. (eds.), Les violences rurales au quotidien, Paris, La Boutique de l'histoire, 1999.

Chauvaud F., Vernois S. (eds.), La justice en images, Paris, CREDHESS, 2004 (Sociétés et représentations 18).

Coll., La statistique judiciaire; son histoire et ses usages scientifiques, Déviance et société, 1998, $22-2$.

Corbin A., Le village des cannibales, Paris, Aubier, 1990.

Dauchy S., Humbert S., Royer J.-P. (ed.), Le juge de paix, Villeneuve d'Ascq, Centre d'histoire judiciaire, 1995.

Deak I., GrossJ. T., Judt T.(eds.), The Politics of Retribution in Europe. World War II and Its Aftermath, Princeton, Princeton University Press, 2000.

Delattre S., Les douze heures noires. La nuit à Paris au XIX ${ }^{e}$ siècle, Paris, Albin Michel, 2000. 
Delpal B., Faure O. (eds), Religion et enfermements (XVII $-\mathrm{XX}^{e}$ siècles), Rennes, Presses Universitaires de Rennes, 2005.

Dumont J.-N., Truche P. (ed.), Histoire et justice. Peut-on juger l'histoire ?, Lyon, Le collège supérieur, 2002.

Ebel É., Les préfets et le maintien de l'ordre public, en France au XIX siècle, Paris, La Documentation Française, 1999.

Ebel É., Police et société : Histoire de la police et de son activité en Alsace au XIXe siècle, Strasbourg, Presses Universitaires de Strasbourg, 2000.

Emsley C., Gendarmes and the State in Nineteenth-Century Europe, Oxford, Oxford University Press, 1999.

Farcy J.-C., Les camps de concentration français de la Première Guerre mondiale (1914-1920), Paris, Anthropos-Economica, 1995 (Anthropos Historiques).

Farcy J.-C., La gendarmerie, police judiciaire au XIX siècle, dans Histoire, Économie, Société, 2001, 20-3, pp. 385-403.

Farcy J.-C., Les rapports des procureurs généraux de la cour d'appel de Dijon (décembre 1849-juillet 1870), Dijon, Éditions universitaire de Dijon, 2003.

Farcy J.-C., La jeunesse rurale dans la France du XIX $X^{e}$ siècle, Paris, Christian, 2004 (Vivre l'histoire).

Fecteau J.-M., La liberté du pauvre. Crime et pauvreté au XIXe siècle québécois, Montréal, VLB éditeur, 2004.

Gacon S., L'amnistie. De la commune à la guerre d'Algérie, Paris, Seuil, 2002.

Horne J., Kramer A., German Atrocities, 1914. A History of Denial, New Haven et Londres, Yale University Press, 2001, trad. française, 1914, Les atrocités allemandes, Paris, Tallandier, 2005.

Kalifa D., L'encre et le sang. Récits de crime et société à la Belle Époque, Paris, Fayard, 1995.

Kalifa D., Naissance de la police privée. Détectives et agences de recherches en France (1832-1942), Paris, Plon, 2000.

Kalifa D., Crime et culture au XIX ${ }^{e}$ siècle, Paris, Perrin 2005 (Pour l'histoire).

Kaluszynski M., La République à l'épreuve du crime : la construction du crime comme objet politique, 1880-1920, Paris, L.G.D.J., 2002.

Krakovitch O., Les femmes bagnardes, Paris, Olivier Orban, 1980.

L'épuration de la magistrature de la Révolution à la Libération, Paris, Loysel, 1994 (Histoire de la justice $\left.n^{\circ} 6\right)$.

La Gendarmerie. De La Révolution à l'entre-deux-guerres, Revue de la gendarmerie nationale, Hors série, 1991.

La Gendarmerie. De l'entre-deux-guerres aux années soixante, Revue de la gendarmerie nationale, Hors-série $n^{\circ} 3,3^{\mathrm{e}}$ trim. 2002.

La justice des années sombres 1940-1944, Paris, La Documentation française, Paris, 2001.

Le Naour J.-Y., Misères et tourments de la chair durant la Grande Guerre, Paris, Aubier, 2002.

Le Naour J.-Y., La honte noire, l'Allemagne et les troupes coloniales françaises, 1914-1945, Paris, Hachette, 2003. 
Lenoir R., Tsikounas M., Yvorel J.-J., Michel Foucault. Surveiller et punir : la prison vingt ans après, CREDHESS, Paris, 1996 (Sociétés et représentations).

Les archives contemporaines à l'administration centrale. Guide de recherches, Paris, ministère de la Justice, 1997.

L'épuration de la magistrature de la Révolution à la Libération : 150 ans d'histoire judiciaire, Paris, 1994 (Histoire de la Justice $n^{\circ}$ 6).

Lessellier C., Les femmes et la prison 1815-1839, Paris, Université de Paris VII, 1982 (Thèse de III ${ }^{\mathrm{e}}$ cycle en Histoire).

Lévy R., Police and the Judiciary in France since the Nineteenth Century, British Journal of Criminology, 1993, 33-2, pp.167-186.

Luc J.-N. (dir.), Gendarmerie, État et société au XIX' siècle, Paris, Publications de la Sorbonne, 2002. Luc J.-N. (dir.), Figures de gendarmes, Paris, CREDHESS, 2003 (Sociétés et représentations, 16). Luc J.-N. (dir.), Histoire de la maréchaussée et de la gendarmerie. Guide de recherche, Maisons-Alfort, Service historique de la gendarmerie nationale, 2005.

Martin B. F., Crime and Criminal Justice Under the Third Republic. The Shame of Marianne, Baton Rouge-Londres, Louisiana State University Press, 1990.

Meinen I., Wehrmacht et prostitution sous l'Occupation 1940-1945, Paris-Genève, Payot, 2006.

Miquel P., Les gendarmes, Paris, Olivier Orben, 1991.

Miquel P., La main courante. Les archives indiscrètes de la police parisienne 1900-1945, Paris, Albin Michel, 1997.

Mucchielli L., Histoire de la criminologie française, Paris, L'Harmattan, 1994.

Offenstadt N., Les fusillés de la Grande guerre et la mémoire collective (1914-1999), Paris, Odile Jacob, 1999.

Olivier C., Le vice et la vertu. Vichy et les politiques de la sexualité, Toulouse, Presses Universitaires du Mirail, 2005.

Ordre public et histoire. La naissance d'une mémoire, Les cahiers de la sécurité intérieure, $3^{\mathrm{e}}$ trim. $1994, \mathrm{n}^{\circ} 17$.

Pierre P., La prison sous Vichy, Paris, Les Éditions de l'Atelier, 1993.

Perrot M., Les ombres de l'histoire. Crime et châtiment au XIX siècle, Paris, Flammarion, 2001.

Peschanski D., La France des camps. L'internement 1938-1946, Paris, Gallimard, 2002.

Petit J.-G., Une justice de proximité : la justice de paix 1790-1958, Paris, PUF, 2003.

Pierre M., Bagnards, la terre de la grande punition : Cayenne 1852-1953, Paris, Autrement, 2000.

Ploux F., Guerres paysannes en Quercy. Violences, conciliations et répression pénale dans les campagnes $d u$ Lot (1810-1860), Paris, Boutique de l'Histoire, 2002.

Renneville M., Le langage des crânes. Une histoire de la phrénologie, Paris, Les empêcheurs de penser en rond, 2000.

Renneville M., Crime et folie. Deux siècles d'enquêtes médicales et judiciaires, Paris, Fayard, 2003.

Rouet G., Justice et justiciables aux XIX et XX $X^{e}$ siècles, Paris, Belin, 1999. 
Rouquet F., L'épuration dans l'administration française : agents de l'État et collaboration ordinaire, Paris, CNRS Éditions, 1993.

Rouquet F., Voldman D., (dir.), Identités féminines et violences politique (1936-1946), Paris, IHTP, 1995 (Les cahiers de l'IHTP, $\mathrm{n}^{\circ} 31$ ).

Shapiro A.-L., Breaking the Codes. Female criminality in Fin-de-Siècle Paris, Stanford, Stanford University Press, 1996.

Sohn A.-M., Chrysalides. Femmes dans la vie privée (XIX'-XXe siècles), Paris, Publications de la Sorbonne, 1996a.

Sohn A.-M., Du premier baiser à l'alcôve. La sexualité des Français au quotidien (1850-1950), Paris, Aubier, 1996b (Collection historique).

Thénault S., Une drôle de justice. Les magistrats dans la guerre d'Algérie, Paris, La Découverte, 2001 (L'espace de l'histoire).

Tillier A., Des criminelles au village. Femmes infanticides en Bretagne (1825-1865), Rennes, Presses Universitaires de Rennes, 2001.

Vimont J.-C., La prison politique en France. Genèse d'un mode d'incarcération spécifique XVIII ${ }^{e}-X^{e}$ siècles, Paris, Anthropos-Economica, 1993 (Anthropos Historiques).

Virgili F., La France « virile ». Des femmes tondues à la libération, Paris, Payot, 2000.

Vogel M., Les polices des villes entre le local et le national. L'administration des polices urbaines sous la III République, Grenoble, Université de Grenoble, 1993 (thèse de doctorat).

Vogel M., Police et espace urbain : Grenoble, 1880-1930, Revue d'histoire moderne et contemporaine, 2003, 50-1, pp. 126-144.

Wagniart J.-F., Le vagabond à la fin du XIXe siècle, Paris, Belin, 1999.

\section{Espaces coloniaux}

Bernault F., Enfermement, prison et châtiments en Afrique du XIX ${ }^{e}$ siècle à nos jours, Paris, Karthala, 1999.

Bernault F., Boilley P., Thioub I. (dir.), Pour l'histoire du contrôle social dans les mondes coloniaux : justice, prisons, et enfermement de l'espace, Outremer, revue d'histoire, 1999, $\mathrm{n}^{\circ}$ 324-325.

Taraud C., La prostitution coloniale. Algérie, Tunisie et au Maroc (1830 à 1962), Paris, Payot, 2003.

\section{NOTES}

1. Lévy (1996, pp. 99-100).

2. Farcy (2001); Lévy (1996), Audren (1996); Chauvaud, Petit (1998).

3. Farcy (2001, pp. 453-460).

4. Voir Rousseaux X., Historiographie du crime et de la justice criminelle dans l'espace français(1990-2005). (I) : du moyen âge à la fin de l'Ancien Régime, Crime, Histoire et Sociétés, 2006, 10, 1, pp. 123-158.

5. Maza (1997, pp. 14-15).

6. Maza (1997, p. 298). 
7. Justice et République(s) (1993); Dauchy, Humbert, Royer (1995), Justice et Institutions (1996); Influences (1999).

8. Rousseaux, Dupont-Bouchat, Vael (1999); Dupont-Bouchat, Rousseaux (1998).

9. de Mari (2000, p. 233).

10. de Mari (2000, p. 252).

11. de Mari (2000, p. 255).

12. de Mari, É., Notes sur la loi du 22 prairial an II et la Grande Terreur, injustice et politique : la Terreur dans la Révolution française, Toulouse, Presses de l'Université des Sciences Sociales de Toulouse, 1997, pp. 69-80.

13. Gueniffey (2000, p. 226).

14. de Mari (2001).

15. Allen (2005, p. 269).

16. Martin (2006, p. 308).

17. Deyon, P. Le temps des prisons. Essai sur l'histoire de la délinquance et les origines du système pénitentiaire, Paris, Éditions universitaires (1975); Foucault M., Surveiller et punir. Naissance de la prison, Paris, Gallimard (1975).

18. Petit (1990, p. 548).

19. Zysberg, A., Les galériens. Vies et destins de 60000 forçats sur les galères de France, 1680-1748 (Paris, 1987); Howard (1994); Vimont (1993); Pierre, M., La terre de la grande punition, Paris, Ramsay, 1981; Pierre (2000).

20. Petit (1990, p. 544).

21. Petit (1990, p. 546).

22. Petit (1990, p. 548).

23. Lessellier (1982).

24. Vimont (1993).

25. Badinter (1992).

26. Joannic-Seta (2000, p. 8).

27. Rappaport (2006).

28. Dupont-Bouchat, La prison pénale, modèles et pratiques. "Révolution ou "Évolution »?

(1775-1815) in Rousseaux, Dupont-Bouchat, Vael (1999, pp. 261-282); Spierenburg (1991).

29. Chauvaud, Petit (1998).

30. Chauvaud, Petit (1998, p. 478).

31. Mucchielli (1994).

32. Perrot (2001).

33. Chauvaud (2000, p. 233; 2003).

34. Chauvaud (2000, p. 15).

35. On retrouve ici, approfondi et discuté le paradigme indiciaire détecté par Carlo Ginzburg, Mythes, emblèmes, traces. Morphologie et histoire, Paris, Flammarion, 1989.

36. Renneville (2000).

37. Renneville (2003, p. 414).

38. Kalifa (1995, p. 10).

39. Kalifa (1995, p. 13).

40. Kalifa (1995, p. 12).

41. Kalifa (1995, p. 302).

42. Kalifa (1995, p. 302).

43. Kalifa (1995, pp. 303-304).

44. Artières (1999, p. 32).

45. Artières (1999, p. 9).

46. Le seul à posséder un visage, via une photographie.

47. Artières (1999, p. 424). 
48. Chauvaud, Vernois (2004); Luc (2003).

49. Kalifa (2005).

50. Introduction à l'Annuaire statistique de la justice, in Rouet (1999, p. 33).

51. Rouet (1999, p. 357).

52. Notamment les nombreuses recherches menées par Bruno Aubusson de Cavarlay (2001), Michelle Perrot ou Philippe Robert ou encore Chauvaud (1995) et La statistique judiciaire (1998).

53. Petit (2003, p. 331).

54. Arnaud-Duc (1997, p. 28).

55. Arnaud-Duc (1997, p. 215).

56. Arnaud-Duc (1997, p. 216).

57. Arnaud-Duc (1997, p. 219).

58. Arnaud-Duc (1997, pp. 220-221). Voir Vesentini (2005).

59. Wagniart (1999, p.10).

60. Wagniart (1999, p. 262).

61. Dupont-Bouchat, Pierre (2001).

62. Kaluszynski (2002).

63. Martin (1990); Allinne (2003-2004).

64. Tillier (2001, p. 12).

65. Lapalus (2004, p. 17).

66. Lapalus (2004, p. 539).

67. Chauvaud (1999, p. 328).

68. Ploux (2002, p. 357).

69. Ploux (2002, p. 359).

70. Corbin (1990, p. 8).

71. Chauvaud, Mayaud (2005).

72. Farcy (2004).

73. Caron (1999).

74. Référence est faite ici à l'œuvre magistrale de Weber E., From Peasants to Frenchmen: The Modernization of Rural France 1870-1914, Stanford, Stanford University Press (1976); La fin des terroirs. La modernisation de la France rurale 1870-1914, Paris, Fayard (1983).

75. Bouchet, Leggett, Vigreux, Verdo (2005, p. 270).

76. Bourdin, Caron, Bernard (2005).

77. Farcy (2001); Shapiro (1996).

78. Revenin (2005).

79. Revenin (2005, p. 167).

80. Delattre (2000, p. 324).

81. Delattre (2000, p. 432).

82. Delattre (2000, p. 554).

83. Perrot, ouverture in Bard, Chauvaud, Perrot, Petit (2002).

84. Bourguinat (2002).

85. Dupont-Bouchat, Pierre (2001).

86. Shapiro (1996).

87. Sohn (1996b).

88. Sohn (1996b, p. 10).

89. Sohn (1996b, p. 310).

90. Sohn (1996b, p. 308).

91. Ebel (1999).

92. Farcy (2003, p. 8).

93. Milliot (2006).

94. Lignereux (2002). 
95. Lévy, R., Les polices aux XIX et XX ${ }^{\mathrm{e}}$ siècles, in Ordre public et histoire (1994, p. 23).

96. Berlière (1993, pp. 20-21).

97. Benabou (1987).

98. Lévy, R., Les polices aux XIX et XX ${ }^{\mathrm{e}}$ siècles, in Ordre public et histoire (1994, p. 25).

99. Lévy, R., Les polices aux XIX et XX ${ }^{\mathrm{e}}$ siècles, in Ordre public et histoire (1994, p. 24); Vogel (1993).

100. Vogel (2003, p. 144).

101. Kalifa (2000, p. 19).

102. Lorgnier (1994).

103. Voir Lévy, R., Les polices aux XIX et $X^{\mathrm{e}}{ }^{\mathrm{e}}$ siècles, in Ordre public et histoire (1994, p. 26).

104. Sur l'Alsace, Ebel (2000).

105. La gendarmerie $(1991 ; 2002)$.

106. Luc (2002).

107. Luc (2005).

108. Luc (2003).

109. Miquel (1997, p. 28). Il en va de même d'un autre ouvrage consacré par l'auteur aux forces de l'ordre, Miquel (1991).

110. Petit, Castan, Faugeron, Pierre, Zysberg (1991).

111. Perrot (2001).

112. Par exemple le colloque de Vaucresson : Michel Foucault (1996).

113. Vimont (1993).

114. Pierre (2000); Krakovitch (1990).

115. Perrot (2001), recueille les principaux articles de l'auteur sur la prison.

116. Badinter (1992, p. 388).

117. Badinter (1992, p. 391).

118. Pierre (1981).

119. Forlivesi, Pottier, Chassat (2005).

120. Pierre E., La colonie de Mettray, in Forlivesi, Pottier, Chassat (2005, p. 49).

121. Delpal, Faure (2005).

122. Berlière, Chabrun (2001, p. 19).

123. Berlière, Chabrun (2001, p. 350).

124. Baruch, Duclerc (2002, p. 12).

125. Gacon (2002).

126. Bancaud (1993).

127. L'épuration (1994).

128. Thénault (2001, p. 9).

129. Thénault (2001, p.11).

130. Thénault (2001, p. 320).

131. Peschanski (2002, p. 17).

132. Farcy (1995, pp. 360, 364).

133. Peschanski (2002, p. 23).

134. Peschanski (2002, pp. 475 et ss.). Voir également Pedron (1993).

135. Aubusson de Cavarlay, Huré, Pottier (1993, p. 7).

136. Aubusson de Cavarlay, Huré, Pottier (1993, p. 82).

137. Aubusson de Cavarlay, Huré, Pottier (1993, p. 97).

138. Le Naour, Valensi (2003, pp. 12-13).

139. Audoin-Rouzeau (1995, p. 181).

140. Amours que l'on retrouve stigmatisés par l'enfermement des filles suspectes dans les camps (Farcy, 2003).

141. Boninchi (2005).

142. Olivier (2005). 
143. Olivier (2005, p. 23).

144. Rouquet, Voldman (1995).

145. Horne, Kramer (2001, 2005).

146. Offenstadt (1999)

147. Virgili (2001, p. 8).

148. Baruch ( 2003) plaide pour une histoire sociale de l'épuration.

149. Virgili (2001); Meinen (2006)

150. Dhondt, S, Huyse L., La répression des collaborations; (1942-1952); un passé toujours présent, Bruxelles, CRISP, 1993.

151. Bergère (2004, p.15).

152. Rouquet (1993), Baruch (2003).

153. Capdevila (1999), Bergère (2004).

154. Deak, Gross, Judt (2000).

155. Branche (2001, p. 15).

156. Branche (2001, p. 424).

157. Taraud (2003, pp. 9-10).

158. Le Naour (2004).

159. Semelin (2005); Zélis (2005).

160. Le crime et le pouvoir (1999); Dumont, Truche (2002).

161. Farcy (2001, p. 460).

162. Il n'est que de comparer les références invoquées dans les listes H-Net entre H-France et HFrançais.

163. Becker, in Audouin-Rouzeau, Becker, Ingrao, Rousso (2002, p. 339).

\section{AUTEUR}

\section{XAVIER ROUSSEAUX}

FNRS - Université catholique de Louvain, Centre d'Histoire du droit et de la justice, Place Blaise

Pascal, 1 B-1348 Louvain-la-Neuve, rousseaux@chdj.ucl.ac.be 